\title{
Force-induced changes of PilY1 drive surface sensing by Pseudomonas aeruginosa
}

Shanice S. Webster ${ }^{1}$, Marion Mathelié-Guinlet ${ }^{2}$, Andreia F. Verissimo ${ }^{3}$, Daniel Schultz ${ }^{1}$, Albertus Viljoen $^{2}$, Calvin K. Lee ${ }^{4,5,6}$, William C. Schmidt ${ }^{4,5,6}$, Gerard C. L. Wong ${ }^{4,5,6}$, Yves F. Dufrene ${ }^{2}$, George A. O'Toole ${ }^{1, *}$

${ }^{1}$ Department of Microbiology and Immunology, Geisel School of Medicine at Dartmouth, Hanover NH 03755, USA

${ }^{2}$ Louvain Institute of Biomolecular Science and Technology, Université Catholique de Louvain, Croix du Sud, 4-5, bte L7.07.07, B-1348 Louvain-la-Neuve, Belgium

${ }^{3}$ Institute for Biomolecular Targeting (bioMT), Geisel School of Medicine at Dartmouth, Hanover NH 03755, USA

${ }^{4}$ Department of Bioengineering, University of California Los Angeles, CA 90095

${ }^{5}$ Department of Chemistry and Biochemistry, University of California Los Angeles, CA 90095

${ }^{6}$ California NanoSystems Institute, University of California Los Angeles, CA 90095

\section{"Corresponding author:}

George A. O'Toole

Dept. of Microbiology \& Immunology

Rm 202 Remsen Building

2866 College Street

Geisel School of Medicine at Dartmouth

Hanover, NH 03755

Phone: (603) 650-1248

32 georgeo@dartmouth.edu 


\section{Abstract}

During biofilm formation, the opportunistic pathogen Pseudomonas aeruginosa uses its type IV pili (TFP) to sense a surface, eliciting increased second messenger production and regulating target pathways required to adapt to a surface lifestyle. The mechanisms whereby TFP detect surface contact is still poorly understood, although mechanosensing is often invoked with little data supporting this claim. Using a combination of molecular genetics and single cell analysis, with biophysical, biochemical and genomics techniques we show that force-induced changes mediated by the von Willebrand A (vWA) domain-containing, TFP tip-associated protein PilY1 are required for surface sensing. Atomic force microscopy shows that PilY1 can undergo forceinduced, sustained conformational changes akin to those observed for mechanosensitive

51 proteins like titin. We show that mutation of a single cysteine residue in the vWA domain results

52 in modestly lower surface adhesion forces, increased nanospring-like properties, as well as

53 reduced c-di-GMP signaling and biofilm formation. Mutating this cysteine has allowed us to

54 genetically separate TFP function in twitching from surface sensing signaling. The conservation

55 of this Cys residue in all $P$. aeruginosa PA14 strains, and its absence in the $\sim 720$ sequenced

56 strains of $P$. aeruginosa PAO1, could contribute to explaining the observed differences in

57 surface colonization strategies observed for PA14 versus PAO1.

Importance

60 Most bacteria live on abiotic and biotic surfaces in surface-attached communities known as

61 biofilms. Surface sensing and increased levels of the second messenger molecule c-di-GMP are

62 crucial to the transition from planktonic to biofilm growth. The mechanism(s) underlying TFP-

63 mediated surface detection that triggers this c-di-GMP signaling cascade are unclear. Here, we

64 provide a key insight into this question: we show that the eukaryotic-like, vWA domain of the TFP

65 tip-associated protein PilY1 responds to mechanical force, which in turn drives production of a 
66 key second messenger needed to regulate surface behaviors. Our studies highlight a potential

67 mechanism that could account for differing surface colonization strategies.

68

\section{Introduction}

Pseudomonas aeruginosa is a ubiquitously distributed opportunistic pathogen that encounters mechanical forces during surface sensing - a crucial first step for biofilm formation. The type four pili (TFP) motility appendage is integral to surface sensing and is thought to transduce a force-induced signal to the cell interior by detecting the resistance to retraction when cells are surface engaged [1], activating the production of cAMP and c-di-GMP, and regulating target genes that control biofilm formation [2-4]. While the importance of the TFP and its tip associated protein, PilY1, in surface sensing has been proposed, direct evidence of how the TFP/PilY1 sense the surface is lacking. Indeed, much of the supporting evidence of a role for this appendage as a key surface sensor is deductive, or alternatively, rely on biological responses or phenotypic changes that are observed during the switch from planktonic to surface-attached growth. In this study, we thus take a multi-disciplinary approach to investigate the mechanism whereby the TFP via the tip-associated protein, PilY1, is directly involved in surface sensing.

PilY1 is part of the priming complex together with the minor pilins that facilitate incorporation of the PilA subunits into the base of the growing pilus fiber during elongation $[5,6]$. During polymerization, the minor pilins and PilY1 are pushed to the tip of the growing pilus. PilY1 has a C-terminal domain that resembles PilC from Neisseria gonorrhoea and a N-terminal von Willebrand $A$ (vWA) domain (Fig. 1A) that is structurally similar to the $A 2$ domain of the human von Willebrand factor (VWF), a force sensing glycoprotein important in stopping bleeding [4]. The vWA domain of PilY1 has the classical Rossman fold - central beta sheets surrounded by amphipathic alpha helices [7] - and a perfectly conserved metal ion dependent adhesion site (MIDAS) containing the conserved DxSxS...T...F motif [8]. vWA domains have been reported in 
93 TFP-associated proteins from other organisms. For example, the vWA domain of the major pilin

94 in Streptococcus agalactiae is essential for adhesion [9] and the MIDAS motif in the vWA

95 domain of the major pilin in Streptococcus sanguinis has recently been shown to be important in

96 binding to eukaryotic cells [10]. Like the vWF [11], the vWA domain of $P$. aeruginosa PA14 PilY1

97 protein also has a high number of cysteine residues; seven out of the 11 cysteines in PilY1 are

98 in its vWA domain. Interestingly, during vascular damage, when exposed to high shear forces

99 due to blood flow, the vWF transitions from a globular to a stretched conformation [12, 13]. This

100 transition is thought to be mediated by a disulfide bond switch exposing specific sites that allow

101 platelets to bind [14-16]. Thus, vWF cysteine residues, depending on their redox state, are key

102 to force sensing, a property that could be hypothesized for cysteine residues in the vWA of

103 PilY1.

Although PilY1 is known to be important in responding to shear forces and in increasing c-di-GMP levels $[3,17,18]$, the precise role of its vWA domain in surface sensing and c-di-GMP

106 signaling is unclear. Our previous genetic studies show that PilY1 and the vWA domain are

107 important for surface-dependent stimulation of c-di-GMP production $[3,18]$. These studies also

108 showed that while the C-terminal domain of PilY1 was dispensable for surface-dependent c-di-

109 GMP production, strains with mutations in the vWA domain failed to regulate c-di-GMP levels

110 and c-di-GMP-related behaviors [18]. Additionally, deletion of the vWA domain is shown to lock

111 PilY1 in a constitutively active signaling conformation that induces virulence independent of

112 surface attachment [4], suggesting multiple roles for the vWA domain in the surface-attached

113 biology of $P$. aeruginosa.

114 Recent cryo-electron tomography studies show the vWA domain of PilY1 to be situated at

115 the very tip of the pilus fiber [5] indicating that this domain is likely in immediate contact with the

116 surface and therefore could be directly engaged in surface sensing. Based on the similarities

117 between the human vWF and the vWA domain of PilY1, and its importance in downstream c-di- 
118 GMP signaling, we hypothesized that force-induced conformational changes originating from the

119 vWA domain of PilY1 are mediated by conserved cysteine residues within this putative

120 mechanosensing domain, and together these features of PilY1 are critical for surface sensing.

121 We explore these hypotheses here.

\section{Results}

The von Willebrand A (vWA) domain of PilY1 regulates c-di-GMP levels and biofilm

125 formation. To address the role of the vWA domain of PilY1 in surface sensing and c-di-GMP 126 signaling, we made chromosomal deletions that removed a part $\left(\Delta v W A_{p}\right)$ or the full $\left(\Delta v W A_{f}\right.$, Fig.

127 1A) vWA domain, then performed static biofilm assays and measured global levels of c-di-GMP

128 (Fig. 1B and C). Our bulk assays show that both the $\Delta v W A_{p}$ and $\Delta v W A_{f}$ variants resulted in a 129 significant decrease in biofilm formation and reduction in global c-di-GMP levels (as seen for the $130 \Delta v W A_{f}$ variant) as compared to WT. These vWA variants also resulted in no twitching motility

131 (Fig. 1D). To confirm these twitch phenotypes, another more sensitive assay was used based

132 on the lysis of the host cells $P$. aeruginosa PA14 by the lytic DMS $3_{\text {vir }}$ phage, which specifically 133 targets the TFP and also requires retraction of surface-expressed pili for infection [19]. Strains 134 carrying the $\Delta v W A_{p}$ and $\Delta v W A_{f}$ variants showed partial zones of clearing in a phage plaquing 135 assays (Fig. S1A), indicating that these strains retained some TFP function. To further ensure 136 that the decrease in biofilm formation and reduced c-di-GMP levels were not due to protein 137 instability, we examined steady state levels of the vWA variants in whole cell extracts. Both the $138 \Delta v W A_{p}$ and $\Delta v W A_{f}$ PilY1 variants were stable and showed a non-significant reduction in whole 139 cell levels as compared to WT (Fig. 1E). However, little surface pili could be detected in the 140 strains expressing the $\Delta v W A_{p}$ and $\Delta v W A_{f}$ variants (Fig. 1F), which likely explains the lack of full 141 pilus function observed in the twitch assays (Fig. 1D). The presence of plaques (Fig. S1A), 142 however, indicates that there are some surface pili, a finding consistent with our Western blots 143 (Fig. 1F). 

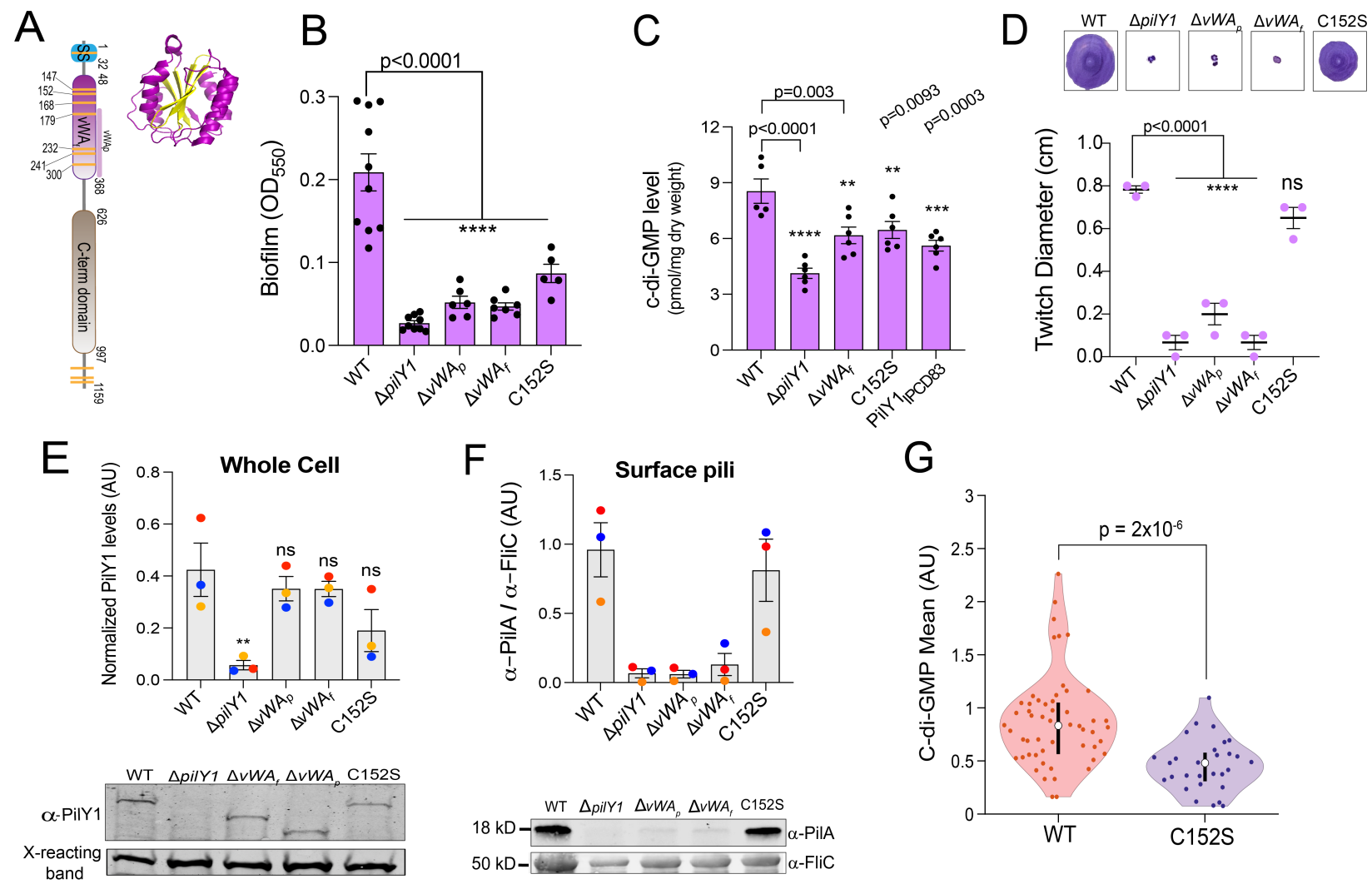

Figure 1. The von Willebrand A (vWA) domain and Cys152 residue of PilY1 are important for regulating c-

di-GMP levels and biofilm formation. A. Schematic showing domain organization of the PilY1 protein. The signal sequence (SS - blue, amino acids 1-32), vWA domain (pink, amino acids 48-368) and C-terminal domain (brown, amino acids 626-997) are highlighted. $\mathrm{vWA}_{\mathrm{p}}$ (amino acids S168-S365) denotes a portion of the vWA domain that is deleted in a mutant analyzed in the subsequent panels. Yellow stripes represent the cysteines residues present in the protein. The vWA domain contains seven of the 11 cysteine residues present in the full length PilY 1 protein with the SS and the C-terminal region having one and three cysteine residues, respectively. Inset: Ribbon diagram showing the vWF A2 domain (PDB 3GXB). The domain shows a classical Rossmann fold [7], comprised of central $\beta$-sheets (yellow) surrounded by $\alpha$-helices (purple). B. Biofilm formation measured at $\mathrm{OD}_{550}$ for WT, the $\triangle$ pilY1 deletion mutant, the vWA variants, and the Cys152S mutant in a static 96 well biofilm assay performed in M8 medium salts plus supplements (see Materials and Methods) and incubated at $37^{\circ} \mathrm{C}$ for $24 \mathrm{~h}$. vWA (amino acids 178-365, see panel A) and $\mathrm{vWA}_{\mathrm{f}}$ indicate a partial and full deletion (amino acids 48-368) of the vWA domain, respectively. Data are from at least five biological replates each with eight technical replicates. C. Quantification of global c-di-GMP levels by mass spectrometry for WT and the indicated mutants shown in picomole per milligram dry weight. Cells were grown on $0.5 \%$ agar plates prepared with M8 medium salts plus supplements, then scraped from the plates after incubation for $37^{\circ} \mathrm{C}$ for $14-16 \mathrm{~h}$. Data are from six biological replicates each with two technical replicates. D. Twitch diameter $(\mathrm{cm})$ for WT and the indicated mutants measured after inoculating LB plates from overnight cultures, then incubating the plates for $24 \mathrm{~h}$ at $37^{\circ} \mathrm{C}$ plus an additional day at room temperature. Representative images of twitch zones are shown above the graph. Data are from three biological replicates. E. Quantification of normalized PilY1 protein levels in whole cell (in arbitrary units (AU)) for WT and the indicated mutants. Cells were sub-cultured from an overnight culture and grown to mid-log phase in M8 medium salts plus supplements and normalized to the same $\mathrm{OD}_{600}$ value. Protein levels in whole cell extracts are normalized to a cross-reacting band at $\sim 60 \mathrm{kDa}$, which is used as an additional loading control. The Cys152S mutant shows a modest but not significant reduction in level in whole cell extracts. A representative Western blot image for PilY1 and the cross-reacting band are shown below the graph. F. Quantification of normalized surface pili levels. PilA ( $\sim 18 \mathrm{kDa})$ protein levels are used as a surrogate for surface pili levels, which are normalized to levels of the flagellar protein, FliC $(\sim 50 \mathrm{kDa})$. A representative Western blot is shown below the graph. All Western blot data are from three biological replicates in three independent experiments. Dots with the same color represent the same biological replicate; different colors indicate different biological replicates. $\mathrm{p}$ values: $\mathrm{p} \leq * 0.05$, ns, not significant. All error bars in Figure 1 are 
standard error of the mean (SEM) and statistical significance was determined by one-way ANOVA and a Dunnett's post-hoc test, p-values: $\mathrm{p} \leq * * * * 0.0001, \mathrm{p} \leq * * * 0.001, \mathrm{p} \leq * * 0.01, \mathrm{~ns}$, not significant. G. Violin plots showing the mean c-di-GMP of the WT strain and a strain expressing the vWA-Cys152S PilY1 variant during early biofilm formation. c-di-GMP level was quantified from GFP intensity determined on a cell-by-cell basis in a microfluidic chamber carrying the $\mathrm{P}_{c d r \mathrm{~A}} \mathrm{GFP}$ construct, which is a reporter of c-di-GMP levels. NOTE: The WT data shown here was first reported in a previous publication [20]; each strain analyses is done independently, in the same system and medium, with the same microscope at identical settings and processed as reported [20]. Given that each analysis is independent but performed identically, we can compare data from previous studies. Each data point represents one tracked cell through an entire division cycle. Statistical significance was determined using the Kruskal-Wallis test, $\mathrm{p}$ $=2 \times 10^{-6}$.
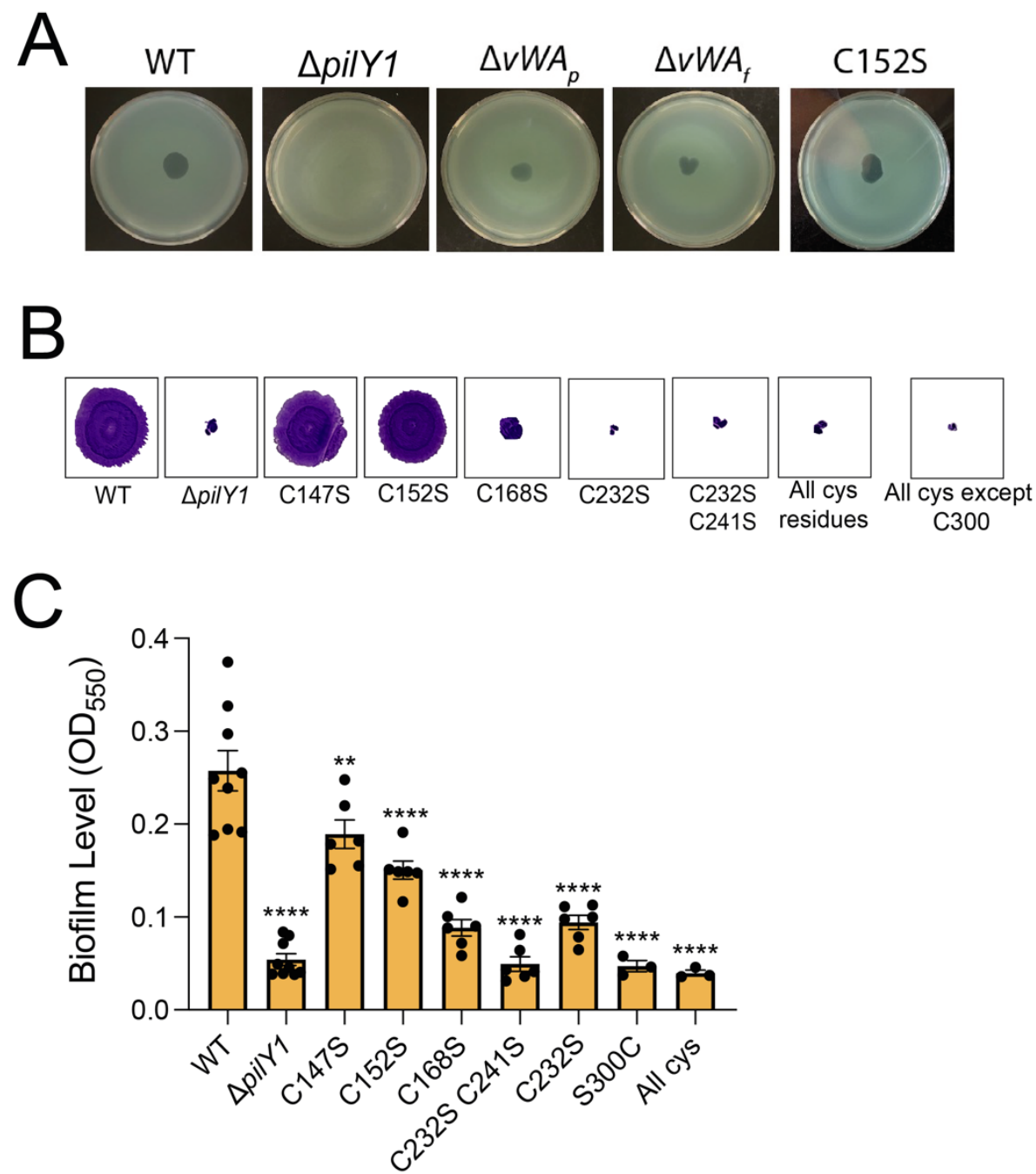

Figure S1. Partial functionality of vWA variants and phenotypic analysis of other cysteine vWA mutants. A. Plaquing assay with phage DMS3 $3_{v i r}$ versus the WT and the indicated mutants as hosts. Zones of clearing shown for WT and the strain expressing the vWA-Cys152S mutant protein are similar, which indicates a similar degree of TFP function. The $\triangle p i l Y 1$ mutant serves as the negative control. B. Representative images of twitch zones stained with crystal violet shown for WT, the $\triangle$ pilY1 or strains expressing PilY1 variants with point mutations in the Cys residues in the vWA domain following incubation at $37^{\circ} \mathrm{C}$ for $24 \mathrm{~h}$ plus one additional day at room temperature. Twitching serves as a measure of TFP function. C. Biofilm level measured at $\mathrm{OD}_{550}$ for WT and the mutants shown in panel B using the 96 well static biofilm assay after $24 \mathrm{hrs}$ at $37{ }^{\circ} \mathrm{C}$, as described in the Materials and Methods. 

in blood clotting by the human vWF are mediated by cysteine residues $[15,16,21]$. Shear

230 forces due to blood flow during vascular damage have been shown to induce disulfide bond

231 cleavage, which results in the protein adopting a new, stretched conformation [21, 22]. Inspired

232 by these studies and the high number of cysteines in the vWA domain of PilY1 (Fig. 1A), we

233 hypothesized that one or more cysteines in the vWA domain of PilY1 might be important for

234 mediating conformational changes in PilY1 and/or the pilus fiber that could in turn impact

235 surface sensing and downstream c-di-GMP signaling. To test this hypothesis, we performed

236 targeted mutagenesis of the cysteine residues in the vWA domain of PilY1 with the aim of

237 identifying one or more of these residues that impact biofilm formation but still retain TFP

238 function as assessed by twitching assays. In all cases, the mutations were introduced into the

239 chromosomal copy of the pilY1 gene, thus the mutants were expressed under the native pilY1

240 promoter and in their native chromosomal context. Of the seven individual and combination

241 cysteine residues mutated, five resulted in decreased biofilm formation but no twitching motility

242 (Fig. S1B and S1C). However, two residues, when mutated (vWA-C147S and vWA-Cys152S)

243 displayed decreased biofilm formation but retained twitching motility (Fig. 1B, D and Fig. S1B).

244 Because the vWA-Cys152S mutation yielded the stronger biofilm phenotype, we focused on this

245 mutant for all subsequent analyses.

246 We next measured c-di-GMP levels globally and on a cell-by-cell basis for the strain

247 expressing the vWA-Cys152S variant. Compared to WT, the vWA-Cys152S mutant showed

248 significantly reduced levels of c-di-GMP based on bulk measurements of cell extracts and on a

249 single-cell basis (Fig. 1C and Fig. 1G, respectively). Note: the WT data shown in the single cell

250 data (Fig. 1G) was first reported in a previous publication [20]. Analyses of WT and vWA-

251 Cys152S were done independently, in the same system and medium, analyzed with the same 
microscope at identical settings and processed as reported [20]. Given that each investigation is independent but performed identically, it allows us to compare data from this previous report. mutant and the WT, we predicted that this point mutation would yield a stable PilY1 protein.

256 Western blot studies of whole cells showed that the vWA-Cys152S variant is stable and shows

257 a modest but non-significant reduction in protein levels as compared to WT PilY1 (Fig. 1E).

258 Additionally, surface pili levels for the strain expressing the vWA-Cys152S mutant protein are

259 comparable to WT (Fig. 1F). These results are consistent with the vWA-Cys152S mutant

260 showing similar levels of twitching motility (Fig. 1D) and plaque formation (Fig. S1A) compared

261 to WT. Of note, none of the observed phenotypes are due to differences in growth rates as the
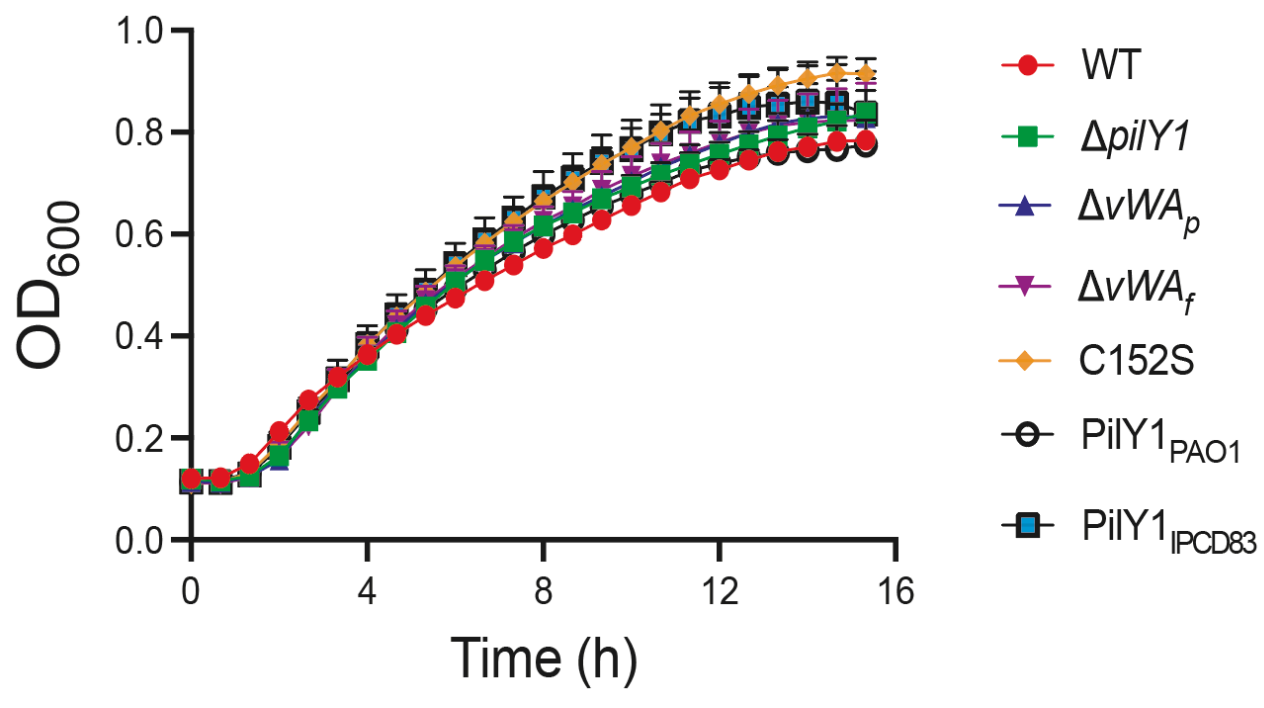

Figure S2. Growth curves for WT and the strains expressing the PilY1 variants. Growth assays were performed in M8 minimal salts medium supplemented with casamino acids, glucose and magnesium sulfate. This medium was also used for all macroscopic biofilm assays, c-di-GMP measurements, plaquing assays and AFM studies. The data are from three biological replicates each with two technical replicates. There is no significant difference among the growth kinetics of each strain. Error bars show SEM and statistical significance was determined at each time point using one-way analysis of variance (ANOVA) using multiple comparisons test. 
273 forces and altered force-induced behaviors. In light of the key role of the vWA domain in

274 biofilm formation and c-di-GMP regulation, we next sought to investigate the different surface

275 adhesion behaviors of $P$. aeruginosa strains expressing WT PilY1, or the PilY1 variants with the

$276 \Delta v W A_{f}$ or the vWA-Cys152S mutations. To this end, we used atomic force microscopy (AFM), a

277 powerful multifunctional technique that has been instrumental in deciphering the adhesion and

278 nanomechanical properties of bacterial pili, at the single-cell and single-molecule levels [23-25].

279 More specifically, we recorded the force experienced by a hydrophobic AFM tip when probed

280 against the TFP of surface engaged bacterial cells as a function of the tip-sample surface

281 distance (Fig. 2A). From the resulting force-distance curves, binding probability and adhesion

282 forces were determined on multiple living cells. As illustrated in the representative force

283 histograms (Fig. 2B), the vWA-Cys152S mutant showed a lower adhesion force than WT cells

$284(\mathrm{~F}=133 \pm 89 \mathrm{pN}$ and $211 \pm 72 \mathrm{pN}$ respectively, $\mathrm{p}<0.001)$, indicating that the Cys $152 \mathrm{~S}$ mutation

285 impacts the interaction strength. However, both WT and vWA-Cys152S PilY1 cells showed a

286 similar binding probability to the hydrophobic AFM tip (Fig. 2C), a result that is consistent with

287 both strains having similar levels of surface pili (Fig. 1G). Cells with the full deletion of the vWA

288 domain $\left(\triangle \mathrm{VWA}_{\mathrm{f}}\right)$ showed an $\sim 0 \%$ binding probability (Fig. 2C) to the hydrophobic tip and a low

289 adhesion force ( 45 pN, Fig. 2B), likely due to a low number of surface pili (Fig. 1F). These

290 data suggest that the observed force curves are dependent on the TFP-associated PilY1.

291 For cells expressing the WT PilY1 and the vWA-Cys152S variant, which both showed

292 adhesion to the hydrophobic AFM tip, two distinct adhesive behaviors were observed, plateaus

293 (red arrow) and spikes (blue arrow; Fig. 2D). Plateaus are defined as adhesive events with a

294 "step" behavior, that is, a constant sustained force over a defined length of time, while spikes

295 are defined as sharp adhesive events with a single minimum and are reflective of a nanospring

296 behavior [25]. Plateaus and spikes are not mutually exclusive in their appearance and 
297 frequency. Cells expressing WT PilY1 or the vWA-Cys152S variant showed plateaus and

298 spikes, however, the frequency of these behaviors differed significantly between the strains

299 (Fig. 2E). Cells expressing the WT PilY1 had a similar proportion of plateaus ( 61\%) and spikes

300 ( $\sim 52 \%$; the sum can be $>100 \%$ because some force curves can have both features). In contrast,

301 cells expressing the vWA-Cys152S mutant of PilY1 showed a significantly lower frequency of

302 plateaus ( $15 \%$ compared to $\sim 61 \%$ for the WT) and a much higher frequency of spikes ( $93 \%$

303 compared to $\sim 52 \%$ for the WT; Fig. 2E). These data indicate that a single point mutation in the

304 PilY1 vWA domain can have a marked impact on the cell's mechanical behavior.

305 Finally, the magnitude of the adhesive signatures for both spikes and plateaus were higher

306 for cells with WT PilY1 than those cells expressing the vWA-Cys152S variant (Fig. 2F and G),

307 consistent with the observation that cells expressing WT PilY1 can sustain globally higher

308 adhesive forces than the cells expressing the vWA-Cys152S mutant (Fig. 2B). Interestingly, for

309 both strains the observed plateau forces are higher than those observed for the spikes, which,

310 along with the higher frequency of plateaus observed in WT PilY1, also explains the higher forces

311 sustained by the WT cells.
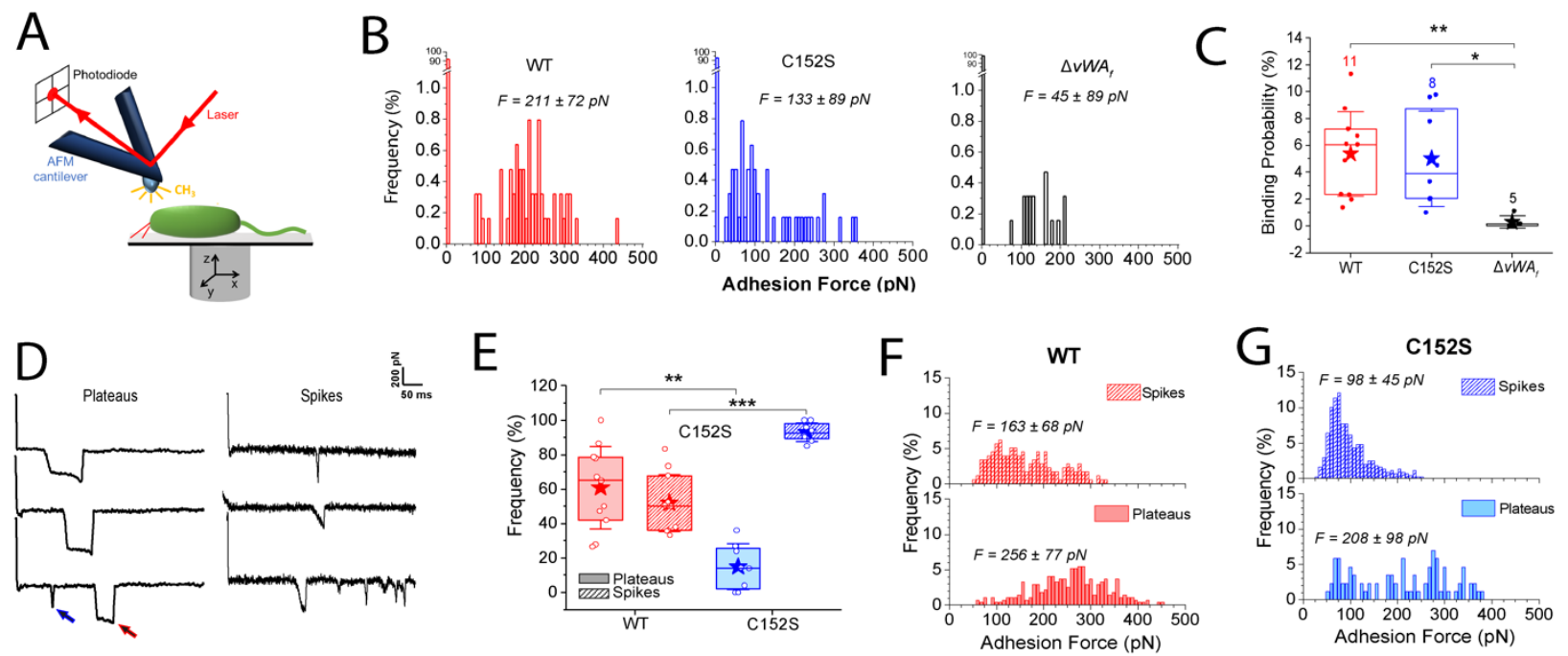

Figure 2. Strains expressing the PilY1-Cys152S mutation display less adhesion force and altered mechanical behaviors compared to strains expressing the WT PilY1. A. Scheme of the AFM setup showing that piliated $P$. aeruginosa is probed with a hydrophobic AFM tip at the free end of the AFM cantilever. Adhesive interactions occurring between the pilus/cell body and the AFM tip cause a deflection of the cantilever, directly proportional to 
Adhesion force histograms between the hydrophobic AFM tip and a representative WT strain, or strains expressing the Cys152S or $\Delta v W A_{f}$ variants of PilY1. For WT: $211 \pm 72 \mathrm{pN}$ ( $\mathrm{n}=55$ adhesive curves); for the vWA-Cys152S: $133 \pm 89 \mathrm{pN}(\mathrm{n}=47)$ and for the $\Delta \mathrm{vWA}_{\mathrm{f}}: 45 \pm 89 \mathrm{pN}(\mathrm{n}=16)$. C. Box plots comparing the binding probability of cells expressing the WT PilY1 or of strains expressing the Cys152S or $\Delta v W A_{f}$ variants of PilY1 are shown. The number of probed cells is indicated. Stars are the mean values, lines the medians, boxes the $25-75 \%$ quartiles and whiskers the standard deviation (SD). Student t-test: $* \mathrm{p} \leq 0.05, * * \mathrm{p} \leq 0.01$. D. Representative retraction force profiles exhibited by the WT or Cys $152 \mathrm{~S}$ mutant cells sorted based on their shape. Plateaus are defined as adhesive events with a "step" behaviour, i.e., a constant sustained force over a defined length of time while spikes are defined as sharp adhesive events with a single minimum. A single retraction profile can feature several plateaus (red arrow), spikes (blue arrow) and even both signatures can occur as marked by the arrows. E. Box plots comparing the occurrence of plateaus (shaded) or spikes (striped) signatures for the WT and Cys152S mutant cells. The number of probed cells is as described in panel C. For the WT, plateaus $=60.8+/-24.0 \%$ and spikes $=51.9+/-16.6 \%, n=11$, and for Cys152S, plateaus $=14.9+/-13.3 \%$ and spikes $=93.1+/-5.4 \%, n=8$. Stars are the mean values, lines the medians, boxes the $25-75 \%$ quartiles and whiskers the SD. Student t-test: ** $\mathrm{p} \leq 0.01, * * * \mathrm{p} \leq 0.001$. F and $\mathbf{G}$.

Distribution of the adhesion forces exhibited by either the plateaus or the spikes for the WT (F) or the strain carrying the Cys152S mutant of PilY1 (G). The mean values are provided along with the histograms. All data were obtained by recording force-distance curves in medium containing M8 salts with an applied force of $250 \mathrm{pN}$ and a pulling speed of $5 \mu \mathrm{m} / \mathrm{s}$ at room temperature. spike signatures observed for strains expressing WT PilY1 protein versus the vWA-Cys152S mutant protein were dependent PilY1 and its vWA domain. We next asked where these force profiles were dependent of the TFP. Because PilY1 is cell-surface-associated and can be secreted to the cell-surface independent of the TFP machinery [3], we expressed plasmid-borne

341 WT PilY1 and the vWA-Cys152S mutant protein in a $\triangle$ pilA background, lacking the full pilus

342 fiber, and performed AFM experiments. Both strains expressing the WT PilY1 protein and the 343 vWA-Cys152S mutant protein showed little adhesion to the hydrophobic tip (binding probability

$344<1 \%$; Fig. S3A-C). The scarce adhesive events recorded for the strains expressing these

345 proteins in the $\triangle$ pilA background were significantly lower than those exhibited when the pilus

346 was present, and plateau signatures were never observed (Fig. S3D). Instead, typical receptor-

347 ligand signatures were recorded, resembling a spike signature, but with very short rupture

348 length consistent with the length of the protein that is stretched while the AFM tip retracts away

349 from the bacterium (Fig. S3D). Together, the genetic and AFM data support the hypothesis that

350 the adhesive forces measured, as well as the plateaus and spikes signatures exhibited by the 

PilY1 plus the pilus fiber.
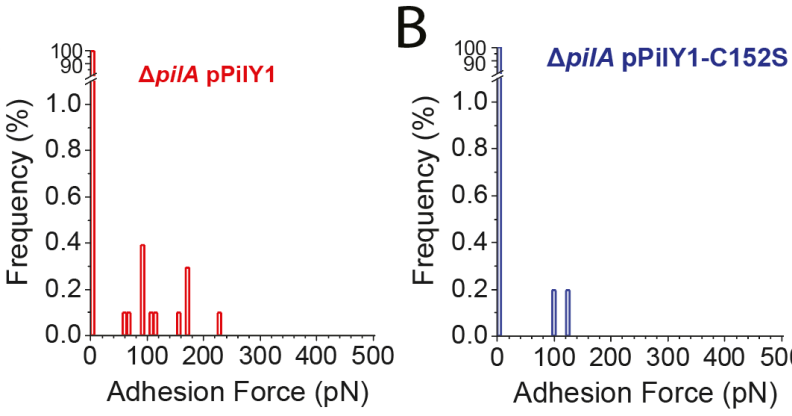

Figure S3. The pilus fiber is required for adhesion to a surface. A-C. Adhesion force histograms obtained by recording force-distance curves between the hydrophobic cantilever tip and representative $\triangle p i l A / \mathrm{pPilY} 1$ (A), $\Delta$ pilA/pPilY1-Cys152S $(\mathbf{B})$ and $\Delta$ pilA/pPilY1- $\Delta \mathrm{vWA}_{\mathrm{f}}(\mathbf{C})$ cell. D. Representative retraction force profiles shown for the same strains.

The vWA-Cys152S mutation has a negligible impact on the solution conformation of the

vWA domain. Given our findings of the significant difference in mechanical behaviors observed

for the strains expressing the WT PilY1 protein and the vWA-Cys152S mutant protein when these strains are engaged with a surface and thus under mechanical tension, we next determined whether this single cysteine mutation affected the conformation of purified, isolated vWA domain of PilY1 in solution. We focused on the vWA domain because despite attempts with several 367 domain of this protein. We cloned WT vWA and vWA-C152S domains (aa 30-369) as glutathione368 S-transferase (GST) fusion proteins to enhance stability and facilitate purification. A GST domain 369 and a HRV-3C protease cleavage site were added to the N-terminus of vWA and the resulting 
370 fusion proteins were overexpressed in E. coli cells and purified to homogeneity (Fig. 3A). The

371 HRV-3C protease cleavage site was confirmed by sequencing. Unfortunately, repeated attempts

372 to efficiently cleave the GST domain from the vWA proteins with protease HRV $3 \mathrm{C}$ were

373 unsuccessful, perhaps due to steric occlusion of the protease binding site in the purified proteins.

374 Thus, the studies below were done using GST-vWA fusion proteins.

375 We performed far-UV circular dichroism (CD) spectroscopy to determine the secondary

376 structure of the WT and mutant fusion proteins and to assess the thermal stability of WT-vWA

377 and the vWA-Cys152S variants (Fig. 3B and 3C). Far-UV CD spectra of the GST-WT-vWA and

378 GST-vWA-Cys152S fusion proteins were monitored at wavelength scans between 195 and 250

$379 \mathrm{~nm}$. Both WT and mutant spectra showed the presence of two distinct negative peaks centered

380 at 208 and $222 \mathrm{~nm}$, typical of $\alpha$-helical proteins (Fig. 3B). Overall, the dichroic spectra for GST-

381 WT-vWA and GST-vWA-Cys152S were similar. Measuring CD as a function of temperature can

382 be used to determine the effects of mutations on protein stability. Analysis of the ellipticity curves

383 in the range of 20 to $90^{\circ} \mathrm{C}$ showed the melting temperatures of GST-WT-vWA and GST-vWA-

$384 \mathrm{C} 152 \mathrm{~S}$ fusion variant to be similar (65.8 versus $63.5^{\circ} \mathrm{C}$; Fig. 3C), suggesting that the $\mathrm{C} 152 \mathrm{~S}$

385 mutation did not perturb the secondary structure of the domain in solution (i.e., in the absence of

386 mechanical force).
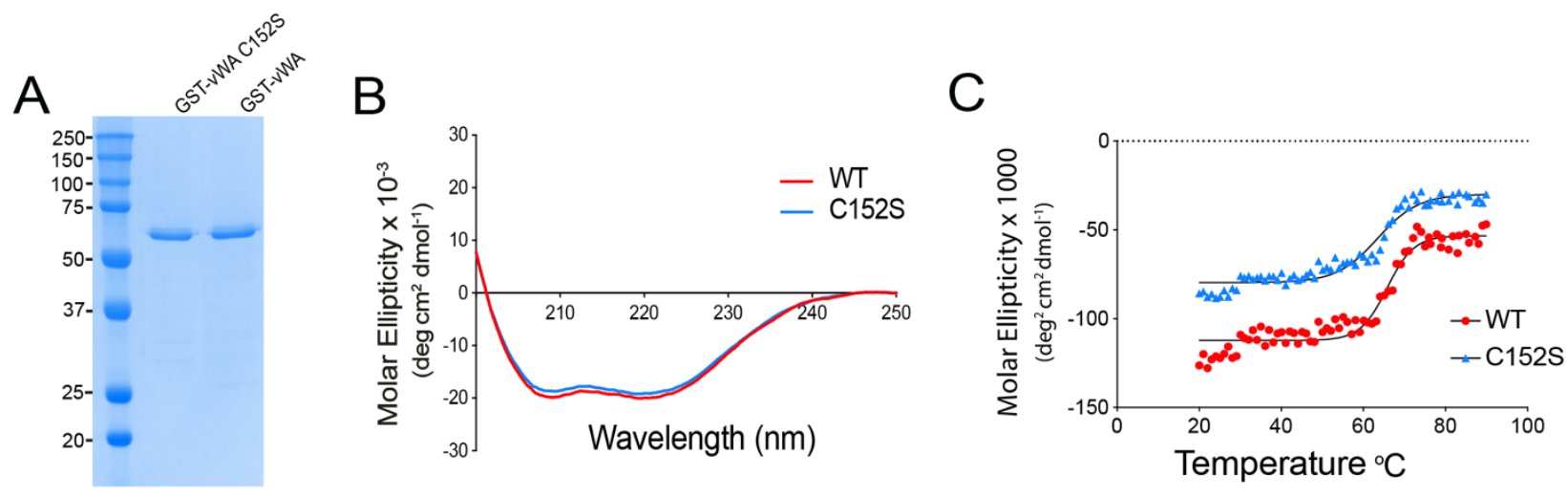

Figure 3. vWA-C152S mutation does not substantially alter conformation of the vWA domain. A. Coomassie 
Methods. The molecular weight markers are indicated. B. Far-UV Circular dichroism (CD) spectra shown in molar ellipticity for the WT GST-vWA (red line) and GST-vWA-C152S mutant (blue line) between 195 and $250 \mathrm{~nm}$ at 20 ${ }^{\circ} \mathrm{C}$. C. Curves of ellipticity at $208 \mathrm{~nm}$ wavelength as a function of temperature for WT and mutant fusion proteins. Spectra were recorded for each sample from 20 to $90^{\circ} \mathrm{C}$ in $1^{\circ}$ increments. Curves were fitted to a Boltzmann sigmoidal equation and the $\mathrm{V}_{50}$ value was determined ( 65.8 versus $63.5^{\circ} \mathrm{C}$ for GST-WT-vWA and GST-vWAC152S fusion variant, respectively).

\section{Genomic analyses reveal that PAO1 strains lack the vWA-C147 and vWA-Cys152 cysteine} residues that are present in PA14 strains, with associated functional consequences.

Given our findings that cells expressing the vWA-Cys152S mutation impact surface sensing, cdi-GMP levels and biofilm formation (Fig. 1B-C, Fig. S1B) in P. aeruginosa PA14, we analyzed whether the Cys 152 residue was conserved across $P$. aeruginosa strains. We leveraged PilY1 sequences from the international $P$. aeruginosa consortium database (IPCD), a repository for thousands of $P$. aeruginosa isolates from a diverse range of environments [26]. We analyzed the phylogenetic relationship of PilY1 amino acid sequences from a total of $852 P$. aeruginosa genomes and found two distinct clades, PA14 (red dot) and PAO1 (dashed circle; Fig. 4A), largely consistent with a previous report by Levesque and colleagues [26]. The PilY1 sequence from the strain, IPCD83 (blue dot), falls within the PAO1 clade. Alignment of the amino acid sequences of the vWA domain of PilY1 from the PA14, PAO1 and the IPCD83 strains show that five of the seven cysteines (magenta) in the vWA domain of PA14 are highly conserved in PAO1 and IPCD83, although the spacing of the residues varies in some cases (Fig. 4B). All three domains consist of positive, negative, polar and hydrophobic amino acids shown in red, blue, green and grey, respectively. Of note is the high abundance of polar residues in the vWA domains of all three strains.

To examine the amino acid diversity of the PilY1 sequences in the PA14 and PAO1 clades, we computed Shannon diversity index as a measure of sequence diversity (Fig. 4C and 4D). We aligned PilY1 sequences within the PA14 (Fig. 4C) and PAO1 (Fig. 4D) clades and calculated Shannon diversity at each amino acid position. 
As shown in Figure 4C, there is very little amino acid sequence diversity over the entire

419 PilY1 sequence among the 129 isolates with PA14 versions of PilY1. Interestingly, all strains in

420 the PA14 clade except one contain the 11 cysteines (magenta circles) found in the PA14

421 reference strain (Fig. 4C). Furthermore, each isolate had all seven cysteines in the vWA domain

422 while there was one strain missing vWA-C232 residue (green circle; a residue we found crititcal

423 for TFP-mediated twitching motility, Fig. S1B). In contrast to the PA14 clade, strains within the

424 PAO1 clade showed low diversity at the C-terminal domain (amino acid 626-997) and high

425 amino acid diversity in the vWA domain (amino acid 48-368; Fig. 4D). Additionally, of the 723

426 variants of the PilY1 sequences from the PAO1 clade analyzed, only eight cysteines were highly

427 conserved compared to the 11 highly conserved cysteines for the PA14 strains. The vWA

428 domain of the PAO1 clade contains five of the seven conserved cysteines found in the PA14

429 clade. Interestingly, vWA-147 and vWA-Cys152 residues are not present in any of the PAO1

430 strains, including the IPCD83 isolate. Recall, that we showed that both vWA-C147 and vWA-

431 Cys152 residues are important in c-di-GMP signaling, and mutations in these residues resulted

432 in strains with decreased biofilm formation but retaining twitching motility (Fig. 1B and Fig.

433 S1B). 


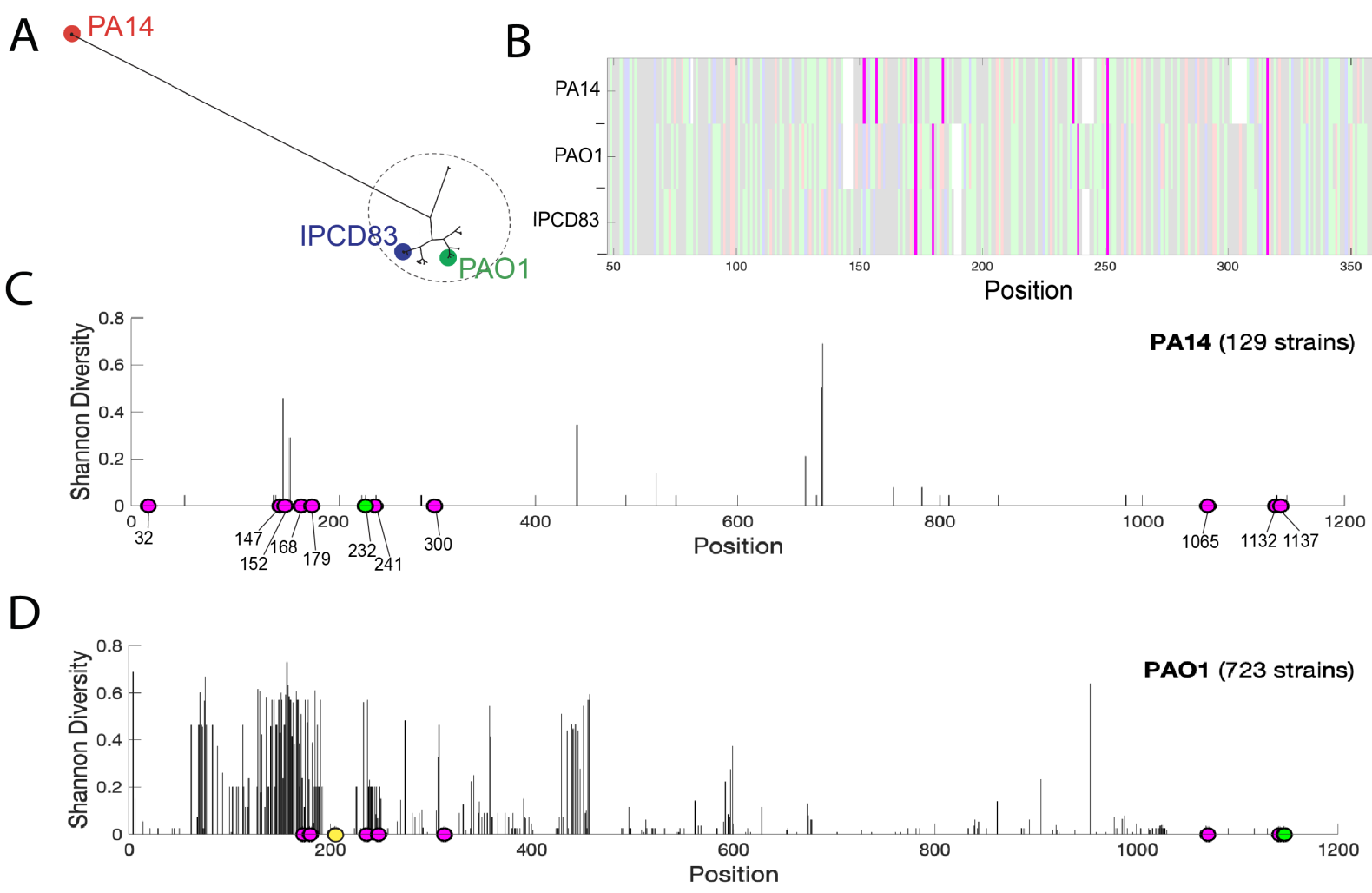

$\mathrm{E}$

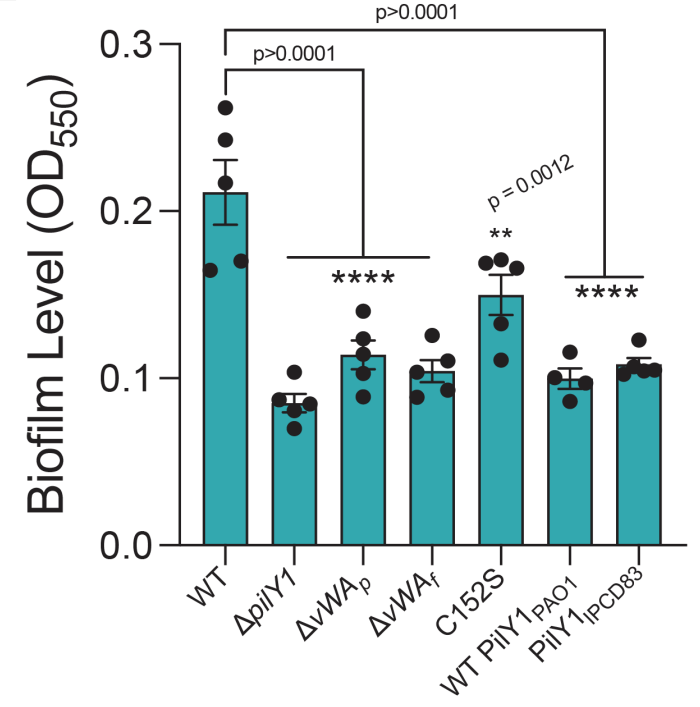

G

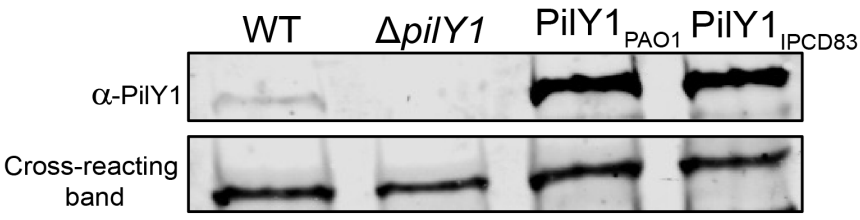

$\mathrm{F}$

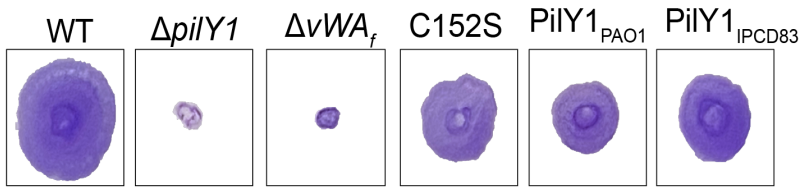

$\mathrm{H}$

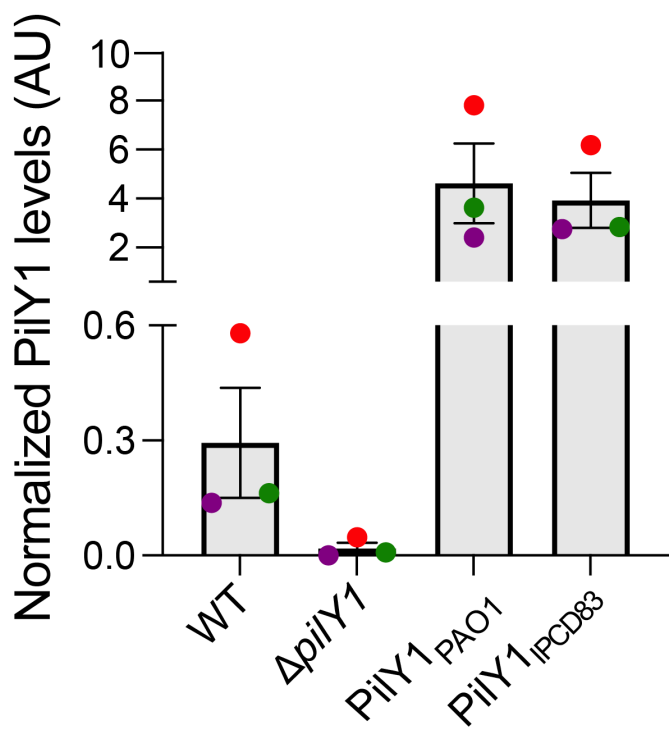


Figure 4. Comparative genomic analyses reveal sequence and functional differences between PA14 and PAO1 alleles of PilY1. A. Phylogenetic tree of PilY1 amino acid sequences obtained from the IPCD database of $P$. aeruginosa genomes [26] showing two distinct clades of PilY1 sequences corresponding to strains from the previously determined P. aeruginosa PA14 and PAO1 clades. The strain labeled IPCD83 is an isolate within the PAO1 clade. B. Alignment of the vWA domain (48 to 368) of PilY1 proteins found in PA14, PAO1 and IPCD83 strains, with cysteines highlighted in magenta. Positive, negative, polar and hydrophobic amino acids are depicted in red, blue, green and grey, respectively. C. Shannon diversity index along the PilY1 amino acid sequence for the 129 PilY1 proteins belonging to the PA14 clade. Fully conserved cysteines are highlighted in magenta. One strain is missing the cysteine depicted in green. D. Shannon diversity index along the PilY1 amino acid sequence for the 723 versions of PilY1 proteins belonging to the PAO1 clade. Fully conserved cysteines are highlighted in magenta. One strain is missing the cysteine depicted in green, and one strain has an extra cysteine depicted in yellow. E. Biofilm formation measured at $\mathrm{OD}_{550}$ in a static 96 well assay for the indicated strains. Hybrid P. aeruginosa PA14 strains carry the PilY1 protein from PAO1 (PilY1 $1_{\mathrm{PAO}}$ ) or the PilY1 protein from IPCD83 strain (PilY1 $1_{\mathrm{IPCD} 83}$ ) replacing the coding region for the $P$. aeruginosa PA14 PilY1 protein. In all cases, the mutant PilY1 variants are expressed from the native locus of $P$. aeruginosa PA14. Error bars are SEM and statistical significance shown was determined by one-way ANOVA and a Dunnett's post-hoc test. p values: $\mathrm{p} \leq * * * * 0.0001, \mathrm{p} \leq * * * 0.001, \mathrm{p} \leq * * 0.01$, ns, not significant. F. Representative images of twitch zones shown for the indicated strains. G. Representative Western blot image for steady state PilY1 protein levels in whole cells WT PilY1, $\Delta$ pilY1, PilY1 from PAO1 (PilY1 $1_{\mathrm{PAO} 1}$ ) and PilY1 variant from strain IPCD83 (PilY1 $1_{\text {IPCD83 }}$. H. Quantification of normalized PilY1 protein levels from whole cells for strains shown in G. Protein level is normalized to a cross-reacting band at $\sim 60 \mathrm{kDa}$. Data are from three biological replicates in three independent experiments. Dots with the same color represent the same biological replicate; different colors indicate different biological replicates.

\section{Given the biofilm phenotype of the strain expressing the vWA-Cys152 variant of PilY1}

(Fig. 1B and Fig. S1B) and the role of PilY1 in early biofilm formation and c-di-GMP signaling, we expected that loss of the vWA-Cys152 residue in strains from the PAO1 clade, including IPCD83, should result in similar phenotypes. To test this hypothesis, we cloned the pilY1 gene from the IPCD83 isolate (PilY1 $1_{\text {IPCD83 }}$ ) or the WT PAO1 strain (WT PilY1 $1_{\text {PAO1 }}$ ) into the native locus of the reference PA14 strain and performed static biofilm assays. Like the vWA-Cys152S mutation, both PAO1 variants expressed in the PA14 strain resulted in significantly decreased levels of biofilm formation as compared to WT (Fig. 4E). Quantification of c-di-GMP levels for

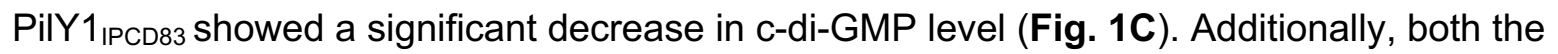
PilY1 $1_{\mathrm{PAO} 1}$ and PilY1 $1_{\mathrm{IPCD} 83}$ variants still supported twitching motility at a level that is similar to the vWA-Cys152S mutant protein (Fig. 4F). The PilY1 $1_{\text {PAO1 }}$ and PilY1 $1_{\text {IPCD83 }}$ variants showed levels of PilY1 expression that exceed the WT (Fig. 4G and Fig. 4H), indicating that the observed phenotypes were not due low-level expression of these variants. 
472

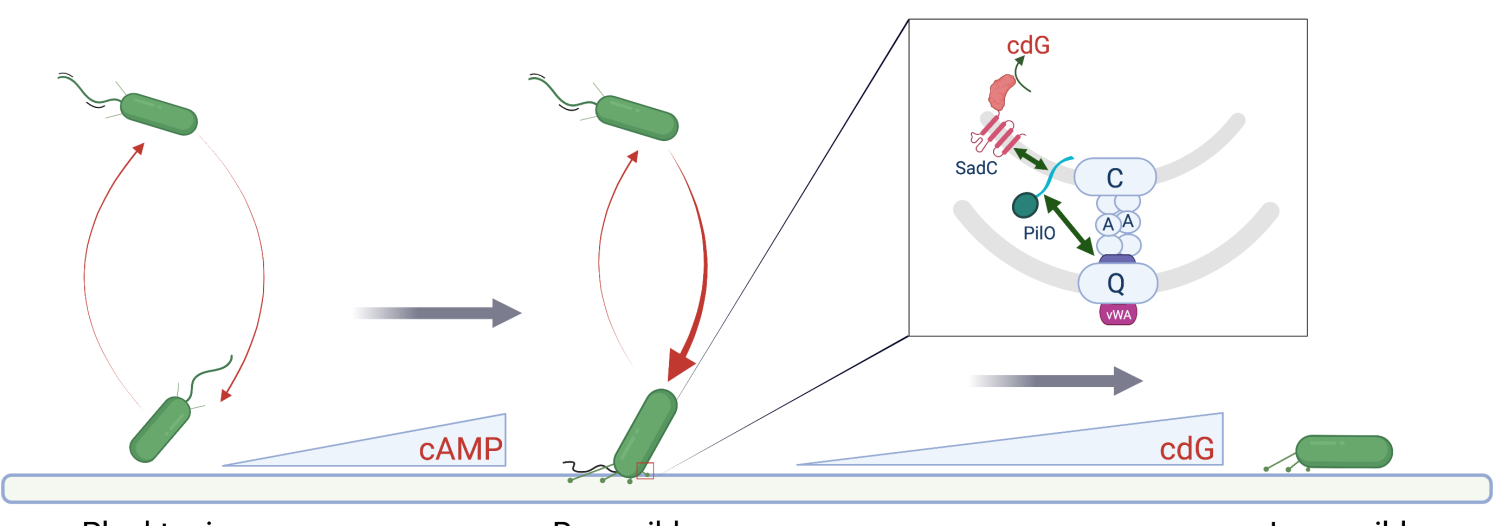

Planktonic
Irreversible

\begin{abstract}
Figure 5. Proposed model for force-induced mechanical force drive transition from planktonic to irreversible attachment. Planktonic bacteria interact with the surface and increase cAMP levels and surface pili levels. The proposed PilY1-PilO interaction can in turn drive the documented PilO-SadC signal transduction cascade which stimulates c-di-GMP signaling and increased biofilm formation.
\end{abstract}

\title{
Discussion
}

Our data show that force-induced changes mediated by one or more cysteine residues in the vWA domain of the TFP tip-associated protein, PilY1, are required for surface sensing and downstream c-di-GMP signaling and biofilm formation. The concept of mechanical force inducing protein conformational changes, that these changes are modulated by disulfide bonds and that such changes in conformation are required for function is well studied in the eukaryotic proteins, titin and vWF. Titin undergoes cycles of folding and refolding that allows it to function as a molecular spring during cycles of muscle relaxation and contraction, respectively [27, 28]. When force is applied, the immunoglobin (Ig) domains of titin unfold and extend [29]. Similarly, increased shear forces due to blood flow cause the vWF to transition from a globular to a stretched conformation [30]; this stretched conformation allows the VWF to bind to platelets and form a clot at sites of vascular damage [31]. Furthermore, the folding and refolding events observed for titin and vWF are mediated by disulfide bonds [32, 33]. For titin, oxidation of the disulfide bond greatly increases both its speed and magnitude of folding [34] while the redox state of the disulfide bond in the A2 domain of the VWF determines exposure of platelet binding 
495 sites [21]. Additionally, disulfides bonds in FimH, the adhesin on the type-I pilus in E. coli [35],

496 are essential for adhesion under high flow environments [36].

497 The vWA domain of PilY1 in P. aeruginosa PA14 has seven cysteine residues. Our

498 genetic analyses show that two of these residues, vWA-Cys152 and to a lesser extent vWA-

499 Cys147, are critical for PilY1-dependent surface signaling and biofilm formation. Our AFM

500 studies support the conclusion that strains expressing the vWA-Cys152S mutant results in cells

501 that are still capable of surface attachment at the same frequency as the WT, and furthermore,

502 this mutation does not destabilize the PilY1 protein. Using AFM, we show that the WT cells

503 display spike signatures, which are typical of nanospring behaviors [25]. That is, T4P/PilY1 can

504 display elastic properties upon the application of force, but once the force is removed, the pilus

505 rapidly returns to its original conformation. Based on previous work [25] and our data here,

506 these force profiles appear to require both TFP and PilY1. Such nanospring properties are also

507 observed for SpaC, a vWA domain-containing protein that is a key pilus-associated adhesin of

508 Lactobacillus rhamnosis GG [23]. Under high mechanical forces, SpaC is shown to behave like

509 a spring. This spring-like behavior is thought to allow the bacterium to withstand higher forces

510 under shear stress when the pilus is stretched, and presumably allow the pilus to engage the

511 surface under strain without snapping [23].

512 The WT P. aeruginosa PA14 strain also shows plateau signatures. One interpretation of

513 these plateau signatures is that they reflect the pilus being bound to the surface at multiple

514 points followed by successive desorption of the pilus [37]. Alternatively, plateaus signatures may

515 be indicative of sustained protein conformational changes. In either case the plateaus observed

516 for WT cells produce high adhesive force signatures, thus likely helping to promote surface

517 engagement.

518 We found that mutating the Cys152 residue of the vWA domain of PilY results in a

519 reduction in biofilm formation and lower levels of c-di-GMP production. A strain expressing this

520 mutant variant also shows significant changes in mechanical properties (detailed below) when 
521 the cell is subjected to force. That these changes in mechanical behavior are dependent on

522 applying a force is in line with our CD and melting curve data which, show no differences in the

523 overall global and secondary structures for WT and the Cys152Ser variant when in solution (i.e.,

524 in the absence of an applied force).

525 The findings from our AFM analysis of the WT and vWA-Cys152Ser allele of PilY1 raise

526 some interesting implications. The $\sim 50-50 \%$ distribution of plateaus and spikes observed in

527 cells with WT PilY1 could suggest a built-in property that allows for inherent heterogeneity in

528 surface adaptation. That is, transient changes in PilY1 conformation (the spike signatures) may

529 not be sufficient to drive signaling; only sustained conformational changes (i.e., plateaus) can do

530 so. Our observation that the vWA-Cys152S mutant variant of PilY1 is skewed 90:10 towards

531 spike signatures (i.e., transient conformational changes), and that this strain is defective for c-di-

532 GMP signalling, supports this conclusion. Thus, not every interaction between a cell and the

533 surface to which it might attach is "productive", a conclusion consistent with several reports

534 showing the heterogenous nature of $P$. aeruginosa populations transitioning to a biofilm lifestyle

$535[20,38-40]$. Furthermore, we could predict then that a PilY1 mutant that favors the plateau

536 conformation should promote c-di-GMP signaling and be a hyper-biofilm former. We have

537 performed extensive genetic screens looking for PilY1 mutants with such phenotypes with no

538 success to-date. Thus, an alternative explanation is that the ability of TFP/PilY1 to transition

539 between conformations is key to the ability to signal properly, and that locking the protein in one

540 conformation, or another, results in aberrant signaling.

541 The critical role for vWA-Cys152 in c-di-GMP signaling and biofilm formation is

542 supported by our genomic analysis, which highlight differences in the PilY1 protein among PA14

543 and PAO1 strains. The vWA domain of PilY1 from the PA14 and PAO1 strains are very

544 different, with PilY1 proteins from the PAO1 clade (PAO1 and IPCD83) lacking the conserved

545 vWA-Cys152 and vWA-Cys147 residues. P. aeruginosa PA14 strains engineered to carry the

546 PAO1 or IPCD83 alleles of PilY1, which lack the conserved vWA-Cys152 and vWA-Cys147 
547 residues, result in a hybrid strain that behaves very much like the $P$. aeruginosa PA14 strain

548 carrying the vWA-Cys152S mutant protein. Thus, our genetic analysis confirmed that the

549 observed sequence differences have functional consequences. The distinct PilY1 proteins of $P$.

550 aeruginosa PA14 and PAO1 may also contribute to explaining the differences in the surface

551 commitment strategies observed for these strains, as reported previously [3, 39].

552 Our AFM data show that force curve plateaus can be maintained for up to $50 \mathrm{~ms}$; it is

553 important to note that this value may be an underestimation because desorption of the pilus

554 from the AFM tip may result in the force curve returning to baseline. With this important caveat

555 in mind and considering that the $P$. aeruginosa TFP has a known retraction rate of $\sim 0.5 \mu \mathrm{m}$

$556 \mathrm{~s}^{-1}[41]$, then the distance that the TFP is retracted during this $50 \mathrm{~ms}$ window (the time spane

557 plateaus are maintained) is $\sim 0.025 \mu \mathrm{m}$. This is quite a short distance (TFP can exceed two

558 microns) and corresponds to the pilus being (almost) fully retracted, with the priming complex

559 (i.e., the minor pilins) and the vWA domain of PilY1 docked into the pore of the secretin [5].

560 Furthermore, if we postulate that TFP/PilY1-mediated signaling is a consequence of mechanical

561 force, for the TFP/PilY1 to remain under force and thus potentially capable of propagating a

562 signaling event via a conformational change, we hypothesize that at least one other pilus would

563 need to be bound to the surface to pull in opposition to the fully retracted pilus described above.

564 That is, PilY1-mediated signaling would require multiple pili to decorate the cell surface - this

565 model has a key corollary in that TFP must be robustly expressed for signaling to occur.

566 Interestingly, previous studies [42-45] and work from our team [3] shows that the level of TFP is

567 low in planktonic cells. Furthermore, our team showed that increased cAMP levels via the Pil-

568 Chp system [3], which is key for pilus production, might require several cellular generations and

569 multiple transient surface interactions to occur [39]. Thus, our previous observations of a role of

570 multigeneration cAMP signaling via TFP may be necessary to produce multiple TFP; multiple

571 TFP, in turn, are required for subsequent c-di-GMP signaling. 
573 39], we propose the following model of the early events initiating biofilm formation by $P$.

574 aeruginosa PA14 (Fig. 5). When the TFP of $P$. aeruginosa PA14 initially engage the surface,

575 we propose that the Pil-Chp signaling cascade promotes cAMP production, which in turn

576 enhances transcription and subsequent production of TFP over the low levels of these

577 appendages produced by planktonically-grown bacteria [3]. Currently, we do not have a strong

578 mechanistic understanding of the linkage(s) among TFP, the Pil-Chp system and cAMP

579 production. However, once more pili are deployed to the surface, this event provides the

580 necessary condition for multiple surface-engaged TFP working in opposition to generate

581 mechanical tension. This mechanical tension in turn can drive the sustained, PilY1-Cys152-

582 dependent conformational changes we have observed for WT cells. That is, the conformational

583 change in vWA domain of PilY1 is maintained only during the application of force when the

584 TFP pull against a solid surface and thereby generate tension (with the cells presumably not

585 moving). We propose that when multiple TFP are engaging the surface, the change in

586 TFP/PilY1 conformation can be sustained as the pilus retracts and PilY1 is docked in the PilQ

587 pore; here PilY1 can interact with PilO, as has been reported for the homologous system in

588 Myxococcus xanthus [5]. Based on our recent study [20], the proposed PilY1-PilO interaction

589 can in turn drive the documented PilO-SadC signal transduction cascade [20], which stimulates

590 c-di-GMP signaling and increased biofilm formation. It is also important to note that a recent

591 pull-down analysis indicate that there is one molecule of PilY1 per pilus in $M$. xanthus [5], thus it

592 is unlikely that intermolecular disulfides are being formed with other PilY1 proteins. Additionally,

593 cryo-electron tomography shows the C-terminal domain of PilY1 to be in direct contact with the

594 minor pilins while the vWA domain is at the apex of the pilus fiber [5], suggesting that

595 intermolecular disulfide bond formation between PilY1 and any of the minor pilins is also

596 unlikely. Consistent with this conclusion, our purification of the vWA domain and Western

597 analysis of cell-surface PilY1 shows no evidence of PilY1 forming intermolecular multimers. 
Finally, our studies were able to successfully separate the role of TFP in motility from its

599 role in signaling. Work from our team and others $[2,3,18,20,46]$ have implicated TFP in

600 surface sensing via the surface-dependent stimulation of the second messengers cAMP and c-

601 di-GMP, however, in these studies the mutants used also disrupted TFP-mediated twitching

602 motility. Here, the Cys152S allele of PilY1 results in a clear loss of c-di-GMP signaling but

603 strains carrying this mutation display robust twitching motility. Together with our previous

604 studies showing a role of the TFP alignment complex component PilO in c-di-GMP production

605 [20], we believe it is quite clear that TFP are not only a key appendage for adhesion and surface 606 motility, but also a central player in surface-specific signal transduction.

\section{Materials and Methods}

609 Bacterial strains, plasmids, media and growth conditions. All bacterial strains used in this

610 study are listed in the supplementary material in Table S1. P. aeruginosa PA14, E. coli S17- $\lambda$-pir

611 were routinely grown in $5 \mathrm{~mL}$ lysogeny broth (LB) medium or struck on $1.5 \%$ LB agar plates with

612 appropriate antibiotics, if necessary. Overnight cultures were grown in LB at 220 rpm on a roller

613 drum. Saccharomyces cerevisiae InvSc1 (Thermo Fisher) used for cloning was maintained on

614 yeast peptone dextrose (YPD - 1\% Bacto yeast extract, 2\% Bacto peptone, and 2\% dextrose)

615 with $2 \%$ agar. Synthetic defined medium without uracil (Sunrise Science Products) was used to

616 select for yeast with construct. All chromosomal point mutation were constructed using the

617 pMQ30 shuttle vector while pMQ72 multi-copy plasmid was used for ectopic expression. All

618 plasmids and oligonucleotides used in this study are listed in Table S2 and Table S3

619 respectively.

621 Construction of deletion mutant strains. All chromosomal in-frame gene deletions were

622 constructed using the pMQ30 shuttle vector carrying the flanking regions of the gene via

623 homologous recombination using the yeast machinery [47] or by Gibson cloning as previously 
624 described in [48]. For yeast cloning, S. cerevisiae was grown overnight at $30^{\circ} \mathrm{C}$ in YPD.

625 Synthetic defined medium without uracil (Sunrise Science Products) was used to select for

626 yeast colonies with the plasmid construct. Plasmids were extracted from yeast using the 'smash

627 and grab' method and transformed by electroporation into E. coli S17 cells and grown on LB

628 plates with $10 \mu \mathrm{g} / \mathrm{ml}$ gentamycin at $30^{\circ} \mathrm{C}$ overnight [2]. Colony polymerase chain reaction

629 (PCR) amplification and sequencing was used to confirm plasmid construction. Plasmids were

630 introduced in $P$. aeruginosa by conjugation and merodiploids were selected on $25 \mu \mathrm{g} / \mathrm{ml}$

631 gentamycin and $20 \mu \mathrm{g} / \mathrm{ml}$ nalidixic acid after which cells were counter-selected on LB with $10 \%$

632 sucrose-containing medium with no added salt [3]. Deletions were confirmed by colony PCR

633 amplification and sequencing with primers flanking the gene. All sequencing was done at the

634 Molecular Biology Core at the Geisel School of Medicine at Dartmouth.

635

636

637

638

639

640

641

642

643

644

645

646

647

648

649

Construction of chromosomal point mutations. Point mutations in the pilY1 gene were made using a modified in vitro site-directed mutagenesis protocol [49]. Forward and reverse complementary primers consisting of the nucleotide codon sequence encoding for the mutation of interest were used to separately amplify the pMQ30 (for chromosomal mutations) or pMQ72 (ectopic expression) parental plasmids with the gene of interest using high fidelity Phusion polymerase (NEB). After four cycles of amplification, the products of these reactions were combined and amplified for an additional 18 cycles with additional Phusion polymerase added.

The parental plasmid was digested for $4 \mathrm{~h}$ using Dpn1 endonuclease (NEB) at $37^{\circ} \mathrm{C}$. Following digestion, the PCR product was transformed into E. coli S17 competent cells and selected on LB with $10 \mu \mathrm{g} / \mathrm{ml}$ gentamycin. Plasmid containing the desired point mutation was isolated and confirmed by sequencing. Introduction of mutations on the chromosome was done by conjugation and counter-selection as described above. All chromosomal mutations were verified by PCR amplification and sequencing. 
650 Biofilm assay. Overnight cultures $(1.5 \mu \mathrm{l})$ were inoculated in U-bottom 96 well plates (Costar)

651 containing $100 \mu \mathrm{M} 8$ salts minimal medium $\left(\mathrm{Na}_{2} \mathrm{HPO}_{4}, \mathrm{KH}_{2} \mathrm{PO}_{4} \mathrm{NaCl}\right)$ supplemented with

652 glucose $(0.2 \% \mathrm{v} / \mathrm{v})$, casamino acids $(0.5 \% \mathrm{v} / \mathrm{v})$ and $\mathrm{MgSO}_{4}(1 \mathrm{mM})$, subsequently referred to as

653 M8 medium. Biofilm assay plates were then stained with $100 \mu \mathrm{l}$ of $0.1 \%$ crystal violet in water

654 for 20 mins at room temperature and destained for 20 mins with $125 \mu$ l de-staining solution

655 (40\% glacial acetic, $40 \%$ methanol and $\left.20 \% \mathrm{H}_{2} \mathrm{O} \mathrm{v} / \mathrm{v}\right)$. Absorbance was read at $\mathrm{OD}_{550}$ and

656 destaining solution was included as the blank. Biofilm assays were done similar to published

657 work by the O'Toole group [50,51].

658

659 In vivo c-di-GMP quantification. Nucleotides were extracted from $P$. aeruginosa cells scraped

660 from $0.52 \%$ agar with $\mathrm{M} 8$ medium after incubation for $37^{\circ} \mathrm{C}$ for $14 \mathrm{~h}$. Cells were removed from

661 plates by gently scraping with a cover slip to avoid scraping the agar, and then immediately

662 placed on ice. Cell pellets were re-suspended in $250 \mu \mathrm{l}$ nucleotide extraction buffer

663 (methanol/acetonitrile/ $\mathrm{dH}_{2} \mathrm{O} 40: 40: 20+0.1 \mathrm{~N}$ formic acid) and incubated at $-20^{\circ} \mathrm{C}$ for $1 \mathrm{~h}$.

664 Following nucleotide extraction, cells were spun for 5 mins at $4^{\circ} \mathrm{C}, 200 \mu \mathrm{l}$ of supernatant was

665 removed and then added to $8 \mu \mathrm{l}$ of $15 \% \mathrm{NH}_{4} \mathrm{HCO}_{3}$ stop solution. Nucleotides were dried in a

666 speed vacuum and resuspended in $200 \mu \mathrm{H}$ HPLC grade water (JT Baker) and placed in screw

667 cap vials (Agilent Technologies). Quantification of c-di-GMP levels was done by liquid

668 chromatography-mass spectrometry (LC-MS/MS) by Lijun Chen at the Mass Spectrometry

669 Facility at Michigan State University. All samples were normalized to dry weight and expressed

670 as $\frac{\text { pmol }}{m g \text { dryweight }}$.

671

672 Macroscopic twitch assay. One percent LB agar plates were stab inoculated using toothpicks

673 dipped in overnight cultures to the bottom of the plate. Plates were incubated at $37^{\circ} \mathrm{C}$ for $24 \mathrm{~h}$

674 and an additional day at room temperature. The agar was subsequently removed from the petri 
675 plate and the twitch zones stained with crystal violet to visualize, and the diameter of the twitch

676 zones measured.

677

678

679

680

681

682

683

684

685

686

687

688

689

690

691

692

693

694

695

696

697

698

699

700

Plaquing assay. One percent agar plates $(60 \times 15 \mathrm{~mm})$ with M8 medium were prepared and cooled to room temperature. Fifty microliters of $P$. aeruginosa overnight culture were added to 1 $\mathrm{mL}$ of $0.5 \%$ warm top agar made with M8 medium and gently mixed. The mixture was quickly poured onto $1 \%$ agar plates made with M8 salts. Plates were swirled to ensure even spreading of top agar. Once cooled, $2 \mu \mathrm{l}$ of phage DMS3 $3_{\text {vir }}$ strain were spotted to the center of the plate, allowed to dry and subsequently incubated at $37^{\circ} \mathrm{C}$ overnight.

Cell surface pili. WT, $\triangle p$ ilY1, vWA variants and vWA-Cys152S cells were streaked in a gridlike pattern on $10 \%$ agar plates with M8 SALTS/supplements and incubated at $37^{\circ} \mathrm{C}$ overnight. Four plates per strain were struck for each biological replicate to ensure adequate number of pili could be recovered. The following day cells were scraped off the plate using a cover slip and put in a $2 \mathrm{~mL}$ tube and vortexed vigorously for 2 mins with $1 \mathrm{~mL}$ of phosphate buffer saline (PBS Corning). Cells suspensions were subsequently spun at $16,000 \times \mathrm{g}$ for 5 mins in a table-top centrifuge and the supernatant removed and transferred to a clean tube and spun again. This step was repeated until no pelleted cells were recovered. Proteins were precipitated with $20 \%$ trichloroacetic acid (TCA - VWR) on ice overnight at $4^{\circ} \mathrm{C}$. Precipitated proteins were collected by centrifuging at $16000 \times \mathrm{g}$ for 25 mins at $4^{\circ} \mathrm{C}$. The supernatant was discarded and the tubes re-centrifuged for 3 mins to get rid of any remaining supernatant. Pellets were washed twice with $1 \mathrm{~mL}$ acetone (VWR) and subsequently air dried to remove residual acetone. Pellets were resuspended in $100 \mathrm{ml}$ 1x sample buffer (BioRad) with b-mercaptoethanol and boiled for 5 mins and then briefly spun before being ran on a $12 \%$ SDS-PAGE gel, and the samples probed for PilA and FliC by Western blot analysis. FliC served as the loading control and was used for normalization of PilA protein levels. Samples were also resolved on a 7.5\% SDS-PAGE gel and 
701 probed for PilY1 using a-PilY1 antibody generously provided by Matt Wolfgang. Western blot

702 analysis was performed as described below.

703

704 Western Blot analysis for PilY1 protein levels in whole cell lysate. All strains were grown

705 overnight in LB at $37^{\circ} \mathrm{C}$. For whole cell lysate (WCL) preps, overnight cultures were diluted

$706 \quad 1: 100$ in $5 \mathrm{~mL}$ M8 SALTS/supplements minimal medium and sub-cultured for $\sim 3 \mathrm{~h}$ at $37^{\circ} \mathrm{C}$.

707 Samples were resolved on a 7.5\% Tris-HCl precast SDS-PAGE gel (Bio-Rad) and blotted onto

$708 \quad 0.45 \mu \mathrm{m}$ pore size nitrocellulose membrane (Bio-Rad) using the $1.5 \mathrm{~mm}$ pre-programmed

709 method on a Trans-Blot Turbo Transfer System (Bio-Rad). The membrane was incubated in

710 blocking buffer (LI-COR Blocking Buffer in TBS) for $1 \mathrm{~h}$ at room temperature and incubated for 1

$711 \mathrm{~h}$ or overnight at $4{ }^{\circ} \mathrm{C}$ in polyclonal a-PilY1 antibody (1:5000 dilution) in BSA TBST $0.1 \%$ buffer.

712 Following incubation with primary antibody, the membrane was washed in TBST $_{0.1 \%}$ for 5 mins

$713 \times 3$ and incubated for $1 \mathrm{~h}$ with goat anti-rabbit in $\mathrm{TBST}_{0.1 \%}(1: 10,000$ dilution) secondary antibody

714 (LI-COR IRDye® 800CW Goat a-rabbit). Incubation with secondary antibody and all subsequent

715 steps were performed in the dark. After incubation with the secondary antibody, the membrane

716 was washed in TBST $_{0.1 \%}$ for 5 mins $x 2$ and then once in TBS. The membrane was imaged using

717 the LI-COR Odyssey CLx imager at BioMT Core at the Geisel School of Medicine at Dartmouth.

718 PilY1 protein levels were quantified relative to the cross-reacting band at $\sim 60 \mathrm{kD}$ using the LI-

719 COR Image Studio Lite software by drawing a rectangle of the same size around each band and

720 using the following background settings: average, border width of 3 , segment $=$ all.

722 Protein quantification. Total protein levels in whole cell lysate was quantified using the Bio-

723 Rad protein assay Dye Reagent as per the manufacturer's instructions as outlined by Bradford

724 [52].

AFM force spectroscopy (AFM). Overnight cultures used in AFM experiments were diluted 200-

727 fold in M8 salts and seeded on hydrophobic non-treated polystyrene petri dishes (Corning) and 
728 left for 10 minutes to adhere [25]. Dishes were then washed gently but thoroughly with M8 salts

729 medium to remove most non-adhered bacteria and used for AFM experiments in the same

730 medium. AFM experiments were performed at room temperature using a NanoWizard® 4

731 NanoScience AFM (JPK Instruments). Gold cantilevers (PNP-TR probes - Pyrex Nitride Probe

732 with Triangular Cantilevers - from NanoWorld) were treated for $16 \mathrm{~h}$ with a $1 \mathrm{mM}$ 1-dodecanethiol

733 solution in ethanol to render them hydrophobic, then rinsed with ethanol and kept in milliQ water

734 until AFM experiments were ready to be performed. Prior any measurements, the cantilever's

735 spring constant was empirically determined by the thermal noise method [53]. The AFM force

736 volume mode was used to record force-distance curve in a pixel-by-pixel manner (force mapping)

737 on $6 \times 6 \mu \mathrm{m}^{2}$ areas $(32 \times 32$ pixels, i.e. 1024 curves) with a bacterium at the center, previously

738 localized by an optical microscope coupled to the AFM. For the $\Delta$ pilA strains overexpressing WT

739 PilY1 or PilY1-Cys152S and lacking the pilus fiber, the area was decreased to $1 \mu m^{2}$ around the

740 bacterium's poles. The following settings were used: an applied force of $250 \mathrm{pN}$, a constant

741 approach/retract speed of $5 \mu \mathrm{m} / \mathrm{s}$ and a z-range of $1.5 \mu \mathrm{m}$.

743 AFM data analysis. Data were analyzed with the data processing software from JPK

744 Instruments (Berlin, Germany). In a first approach, all force distance curves exhibiting an

745 adhesive event were selected, as opposed to the non-adhesive curves which were discarded,

746 thus allowing an estimation of the binding probability. In a second approach, adhesive curves

747 were sorted depending on their signature (plateaus vs spikes) and the maximum adhesion

748 sustained by each adhesive peak was determined. The frequency of plateaus was assessed by

749 dividing the number of curves showing plateaus plus curves showing both plateaus and spikes

750 by the total number of adhesive curves. A similar approach was used to calculate the percent of

751 spikes. The formulas to calculate the percent plateaus $\left(P_{\text {plateaus }}\right)$ or the percent spikes $\left(P_{\text {spikes }}\right)$

752 are shown below: 
(1) $P_{\text {plateaus }}=\left(N_{\text {curves showing only plateaus }}+N_{\text {curves showing plateaus and spikes }}\right) /\left(\mathrm{N}_{\text {curves showing only plateaus }}+\right.$

$$
\mathrm{N}_{\text {curves showing plateaus and spikes }}+\mathrm{N}_{\text {curves showing only spikes) }}
$$

(2) $P_{\text {spikes }}=\left(N_{\text {curves showing only spikes }}+N_{\text {curves showing plateaus and spikes }}\right) /\left(N_{\text {curves showing only plateaus }}+N_{\text {curves }}\right.$

showing plateaus and spikes $+N_{\text {curves showing only spikes) }}$

Shannon Diversity. We performed nucleotide BLAST searches on a local version of the IPCD

761 database of $P$. aeruginosa genomes to identify variants of the PilY1 protein. Using the

762 nucleotide sequences of PilY1 from PA14, PAO1 and IPCD-83 (GenBank: MCMY00000000),

763 we were able to identify 852 strains with versions of the full protein. We used custom MATLAB

764 scripts to perform an alignment of the amino acid sequences of all 852 versions of PilY1 and

765 construct the corresponding phylogenetic tree. We performed the alignment of PilY1 amino acid

766 sequences using a series of BLOSUM80 to BLOSUM30 scoring matrices. We constructed the

767 phylogenetic tree of PilY1 sequences using a Jukes-Cantor maximum likelihood method to

768 estimate the number of substitutions between two sequences and an Unweighted Pair Group

769 Method Average (UPGMA) to construct the phylogenetic tree from the pairwise distances. 129

770 sequences of PilY1 belong to a clade with highly similar proteins, which includes PilY1 from

771 PA14. 723 sequences belong to a diverse clade that includes PilY1 from PAO1 and IPCD-83.

772 Within each of these two groups, we calculated the Shannon diversity in each position along the

773 aligned amino acid sequence using $H=-\sum p_{i} \ln \left(p_{i}\right)$, where $p_{i}$ is the probability of each amino 774 acid (including gaps). Code is available at github.com/GeiselBiofilm. 
776 Growth assays. Overnight cultures were inoculated in M8 salts/supplements at a starting $\mathrm{OD}_{600}$

777 of $\sim 0.05$. Readings were taken every 40 mins for $16 \mathrm{~h}$ in a Synergy Neo2-multimode microplate

778 reader at the BioMT Core at the Geisel School of Medicine at Dartmouth.

780 Cloning and protein expression of GST-vWA fusions. The coding region of the WT and the

781 C152S mutant of the vWA domain (amino acids 30-369) from $P$. aeruginosa PA14-UCBPP were

782 PCR amplified and cloned into pGEX-6p-1 plasmid at the BamHI cut site by Gibson assembly.

783 E. coli BL21 (DE3) competent cells were transformed with plasmid and selected on LB plates

784 with $50 \mu \mathrm{g} / \mathrm{mL}$ carbenicillin grown at $30^{\circ} \mathrm{C}$ overnight. A single colony was used to inoculate 5

$785 \mathrm{~mL}$ of liquid LB and grown for $12-14 \mathrm{~h}$ at $30^{\circ} \mathrm{C}$. Each $5 \mathrm{~mL}$ seed culture was used to inoculate

$500 \mathrm{~mL}$ LB in a $2 \mathrm{~L}$ flask and allowed to grow at $37^{\circ} \mathrm{C}$ with shaking $200 \mathrm{rpm}$ until the $\mathrm{OD}_{600}$ was

0.6-0.8. A total of 6 L LB (12 flasks) were inoculated. Expression was induced with $0.1 \mathrm{mM}$

$\times g$ for $10 \mathrm{~min}$, washed with PBS buffer and stored at $-20^{\circ} \mathrm{C}$ until further use.

Purification of wild-type GST-vWA and Cys152S mutant proteins. E. coli cells expressing

792 WT GST-vWA and GST-vWA-C152S mutant proteins were resuspended in PBS supplemented 793 with $2 \mathrm{mM}$ TCEP (Thermo scientific), $0.01 \mathrm{mg} / \mathrm{mL}$ lysozyme from chicken egg (SIGMA), EDTA-

794 free protease inhibitors cocktail (Blmake) and 10U/mL benzonase nuclease (Millipore) and lysed 795 in a Microfluidizer LM10 (Microfluidics) at 18,000 psi. Nucleic acids were precipitated by addition 796 of $0.1 \%$ polyethylenimine branched (SIGMA). Crude cell lysates were cleared by centrifugation 797 at $200,000 \times g$ for 1 hour at $4{ }^{\circ} \mathrm{C}$ in a Beckman Optima L-70 ultracentrifuge. Clear lysates were 798 incubated overnight with $5 \mathrm{~mL}$ Glutathione Sepharose 4B resin (Cytiva) previously equilibrated 799 with PBS containing 2 mM TCEP. Lysates and resin were transferred to a disposable plastic 800 column and allowed to drain fully (flow through). Resin was washed with at least 15 column 801 volumes of PBS, 2 mM TCEP buffer before elution of the GST-vWA proteins with 5 column 
802 volumes of freshly prepared elution buffer $50 \mathrm{mM}$ Tris $-\mathrm{HCl} \mathrm{pH} 8,10 \mathrm{mM}$ reduced glutathione.

803 Elution fractions were concentrated using 30,000 MWCO $15 \mathrm{~mL}$ Amicon centrifugal filters

804 (Millipore) in a Beckman Allegra 6R centrifuge. Proteins were loaded in a HiLoad Superdex 75

805 pg (Cytiva) pre-packed column equilibrated with $50 \mathrm{mM}$ Tris- $\mathrm{HCl} \mathrm{pH} \mathrm{8,} 150 \mathrm{mM} \mathrm{NaCl}, 1 \mathrm{mM}$

806 TCEP using an AKTApure instrument (Cytiva). Fractions containing the fusion GST-vWA protein

807 were combined and concentrated as before and subjected to a second gel filtration step using a

808 high-resolution Superdex 200 increase 10/300 (Cytiva). Purified WT and C152S mutant GST-

809 vWA proteins were extensively dialyzed against $20 \mathrm{mM}$ sodium phosphate $\mathrm{pH} 7.4$ buffer Final

810 protein concentrations were determined using Bio-Rad protein assay reagent.

811 Circular Dichroism (CD) and melting curves. The far-UV circular dichroism (CD) spectra

812 (195-250 nm) were recorded with a JASCO J-815 spectrophotometer (Jasco, Inc.) equipped

813 with a CDF426S/15 Peltier temperature controller using a 2-mm path length quartz cuvette. CD

814 spectra of proteins were recorded at $20^{\circ} \mathrm{C}$ using a step size of $0.1 \mathrm{~mm}$. A time constant of $12 \mathrm{~s}$

815 was used to improve the signal to noise ratio and to decrease the contribution of the solvent at

816 lower wavelengths. CD spectra were recorded using $1 \mu \mathrm{M}$ of GST-vWA WT and GST-vWA-

817 C152S proteins in $20 \mathrm{~mm}$ sodium phosphate buffer, $\mathrm{pH} 7.4$, and corrected by subtracting the

818 spectrum of the buffer alone.

819 Thermal unfolding curves were obtained by monitoring the ellipticity at 222 and $208 \mathrm{~nm}$

820 of both fusion proteins at $1 \mu \mathrm{M}$ concentration at a heating rate of $1^{\circ} \mathrm{C} \mathrm{min}^{-1}$ in the temperature

821 range of 20 to $90^{\circ} \mathrm{C}$. A 1 s integration time and 5 s equilibration time were used for each

822 measurement and buffer ellipticities at the selected wavelengths were subtracted from the

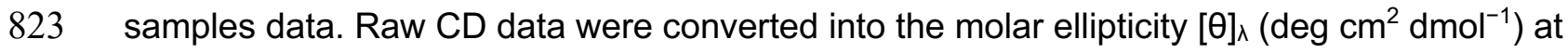

824 each wavelength using the relation, $[\theta]_{\lambda}=\theta_{\lambda} /(10 \mathrm{CNI})$, where $\theta_{\lambda}$ is the observed ellipticity in

825 millidegrees at wavelength $\lambda, C$ is the molar protein concentration, $N$ is the number of amino

826 acids of the protein, and I is the path length of the cuvette in $\mathrm{cm}$. Following CD measurements,

827 protein samples were collected, and protein concentrations measured for accuracy. 
Acknowledgments. We thank Roger Levesque for providing the IPCD strains used in this

830 report and Dr. Sherry Kuchma for building the $\Delta v W A_{p}$ strain. Also, thanks to Dr. Matt Wolfgang

831 for the PilY1 antibody, Emilie Shipman for help with protein purification, Dr. Paul Delfino for

832 technical assistance with CD, Kelsie Leary, Dr. Dean Madden and Dr. Holger Sondermann for

833 advice on analyzing the CD data. The authors would also like to thank rotation students who

834 worked on the project: Alexander Pastora and Rebecca Valls, other members of the O'Toole

835 lab: Chris Geiger, Dr. Sherry Kuchma and Fabrice Jean-Pierre for helpful discussions. This work

836 was supported by the NIH via awards R37 Al83256 (to G.A.O.), R01 Al43730 (to G.C.L.W.) and

837 COBRE/NIGMS 5 P20 GM130454-02 (to D.S). This work was also supported by bioMT through

838 NIH NIGMS grant P20-GM113132. Work at UCLouvain was supported by the Excellence of

839 Science-EOS programme (Grant \#30550343), the European Research Council (ERC) under the

840 European Union's Horizon 2020 research and innovation programme (grant agreement

$841 \mathrm{n}^{\circ} 693630$ ), and the National Fund for Scientific Research (FNRS). Y.D. is a Research Director

842 at the FNRS.

843

844

845

846

847

848

849

850

851

852

853

854

855

856

857

858

859

860

861

862

\section{References}

1. Ellison, C.K., et al., Obstruction of pilus retraction stimulates bacterial surface sensing. Science, 2017. 358(6362): p. 535-538.

2. Persat, A., et al., Type IV pili mechanochemically regulate virulence factors in Pseudomonas aeruginosa. Proc Natl Acad Sci U S A, 2015. 112(24): p. 7563-8.

3. Luo, Y., et al., A hierarchical cascade of second messengers regulates Pseudomonas aeruginosa surface behaviors. mBio, 2015. 6(1).

4. Siryaporn, A., et al., Surface attachment induces Pseudomonas aeruginosa virulence. Proc Natl Acad Sci U S A, 2014. 111(47): p. 16860-5.

5. Treuner-Lange, A., et al., PilY1 and minor pilins form a complex priming the type IVa pilus in Myxococcus xanthus. Nat Commun, 2020. 11(1): p. 5054.

6. Nguyen, Y., et al., Pseudomonas aeruginosa minor pilins prime type IVa pilus assembly and promote surface display of the PilY1 adhesin. J Biol Chem, 2015. 290(1): p. 601-11.

7. Rossmann, M.G., D. Moras, and K.W. Olsen, Chemical and biological evolution of nucleotidebinding protein. Nature, 1974. 250(463): p. 194-9.

8. Lee, J.O., et al., Crystal structure of the A domain from the alpha subunit of integrin CR3 (CD11b/CD18). Cell, 1995. 80(4): p. 631-8. 
863 9. Konto-Ghiorghi, Y., et al., Dual role for pilus in adherence to epithelial cells and biofilm formation

864

865

866

867

868

869

870

871

872

873

874

875

876

877

878

879

880

881

882

883

884

885

886

887

888

889

890

891

892

893

894

895

896

897

898

899

900

901

902

903

904

905

906

907

908

909

in Streptococcus agalactiae. PLoS Pathog, 2009. 5(5): p. e1000422.

10. Raynaud, C., et al., PilB from Streptococcus sanguinis is a bimodular type IV pilin with a direct role in adhesion. Proc Natl Acad Sci U S A, 2021. 118(22).

11. Shapiro, S.E., et al., The von Willebrand factor predicted unpaired cysteines are essential for secretion. J Thromb Haemost, 2014. 12(2): p. 246-54.

12. Schneider, S.W., et al., Shear-induced unfolding triggers adhesion of von Willebrand factor fibers. Proc Natl Acad Sci U S A, 2007. 104(19): p. 7899-903.

13. Zhang, Q., et al., Structural specializations of A2, a force-sensing domain in the ultralarge vascular protein von Willebrand factor. Proc Natl Acad Sci U S A, 2009. 106(23): p. 9226-31.

14. Aponte-Santamaria, C., et al., Force-sensitive autoinhibition of the von Willebrand factor is mediated by interdomain interactions. Biophys J, 2015. 108(9): p. 2312-21.

15. Gogia, S. and S. Neelamegham, Role of fluid shear stress in regulating VWF structure, function and related blood disorders. Biorheology, 2015. 52(5-6): p. 319-35.

16. Purvis, A.R., et al., Two Cys residues essential for von Willebrand factor multimer assembly in the Golgi. Proc Natl Acad Sci U S A, 2007. 104(40): p. 15647-52.

17. Rodesney, C.A., et al., Mechanosensing of shear by Pseudomonas aeruginosa leads to increased levels of the cyclic-di-GMP signal initiating biofilm development. Proc Natl Acad Sci U S A, 2017. 114(23): p. 5906-5911.

18. Kuchma, S.L., et al., Cyclic-di-GMP-mediated repression of swarming motility by Pseudomonas aeruginosa: the pilY1 gene and its impact on surface-associated behaviors. J Bacteriol, 2010. 192(12): p. 2950-64.

19. Cady, K.C., et al., The CRISPR/Cas adaptive immune system of Pseudomonas aeruginosa mediates resistance to naturally occurring and engineered phages. J Bacteriol, 2012. 194(21): p. 5728-38.

20. Webster, S.S., et al., Interaction between the type 4 pili machinery and a diguanylate cyclase fine-tune c-di-GMP levels during early biofilm formation. Proc Natl Acad Sci U S A, 2021. 118(26).

21. Butera, D., et al., Autoregulation of von Willebrand factor function by a disulfide bond switch. Sci Adv, 2018. 4(2): p. eaaq1477.

22. Ganderton, T., et al., Lateral self-association of VWF involves the Cys2431-Cys2453 disulfide/dithiol in the C2 domain. Blood, 2011. 118(19): p. 5312-8.

23. Tripathi, P., et al., Adhesion and nanomechanics of pili from the probiotic Lactobacillus rhamnosus GG. ACS Nano, 2013. 7(4): p. 3685-97.

24. Mignolet, J., et al., AFM Unravels the Unique Adhesion Properties of the Caulobacter Type IVc Pilus Nanomachine. Nano Lett, 2021. 21(7): p. 3075-3082.

25. Beaussart, A., et al., Nanoscale adhesion forces of Pseudomonas aeruginosa type IV Pili. ACS Nano, 2014. 8(10): p. 10723-33.

26. Freschi, L., et al., Clinical utilization of genomics data produced by the international Pseudomonas aeruginosa consortium. Front Microbiol, 2015. 6: p. 1036.

27. Freundt, J.K. and W.A. Linke, Titin as a force-generating muscle protein under regulatory control. J Appl Physiol (1985), 2019. 126(5): p. 1474-1482.

28. Eckels, E.C., et al., The Work of Titin Protein Folding as a Major Driver in Muscle Contraction. Annu Rev Physiol, 2018. 80: p. 327-351.

29. Marszalek, P.E., et al., Mechanical unfolding intermediates in titin modules. Nature, 1999. 402(6757): p. 100-3.

30. Rack, K., et al., Margination and stretching of von Willebrand factor in the blood stream enable adhesion. Sci Rep, 2017. 7(1): p. 14278. 
910 31. Sadler, J.E., Biochemistry and genetics of von Willebrand factor. Annu Rev Biochem, 1998. 67: p.

911 395-424.

912 32. Giganti, D., et al., Disulfide isomerization reactions in titin immunoglobulin domains enable a

913 mode of protein elasticity. Nat Commun, 2018. 9(1): p. 185.

914 33. Marti, T., et al., Identification of disulfide-bridged substructures within human von Willebrand

915 factor. Biochemistry, 1987. 26(25): p. 8099-109.

34. Eckels, E.C., et al., The Mechanical Power of Titin Folding. Cell Rep, 2019. 27(6): p. 1836-1847 e4.

35. Jones, C.H., et al., FimH adhesin of type 1 pili is assembled into a fibrillar tip structure in the Enterobacteriaceae. Proc Natl Acad Sci U S A, 1995. 92(6): p. 2081-5.

919

36. Nilsson, L.M., et al., The cysteine bond in the Escherichia coli FimH adhesin is critical for adhesion

920

921

922

923

924

925

926

927

928

929 under flow conditions. Mol Microbiol, 2007. 65(5): p. 1158-69.

37. Dufrene, Y.F., et al., AFM in cellular and molecular microbiology. Cell Microbiol, 2021. 23(7): p. e13324.

38. Armbruster, C.R., et al., Heterogeneity in surface sensing suggests a division of labor in Pseudomonas aeruginosa populations. Elife, 2019. 8.

39. Lee, C.K., et al., Multigenerational memory and adaptive adhesion in early bacterial biofilm communities. Proc Natl Acad Sci U S A, 2018. 115(17): p. 4471-4476.

40. Kulasekara, B.R., et al., c-di-GMP heterogeneity is generated by the chemotaxis machinery to regulate flagellar motility. Elife, 2013. 2: p. e01402.

930

41. Skerker, J.M. and H.C. Berg, Direct observation of extension and retraction of type IV pili. Proc

931

932 Natl Acad Sci U S A, 2001. 98(12): p. 6901-4.

42. Cowles, K.N. and Z. Gitai, Surface association and the MreB cytoskeleton regulate pilus production, localization and function in Pseudomonas aeruginosa. Mol Microbiol, 2010. 76(6): p.

933

934

935 1411-26.

43. Kelly, N.M., et al., Pseudomonas aeruginosa pili as ligands for nonopsonic phagocytosis by fibronectin-stimulated macrophages. Infect Immun, 1989. 57(12): p. 3841-5.

936

937

44. Speert, D.P., et al., Nonopsonic phagocytosis of nonmucoid Pseudomonas aeruginosa by human neutrophils and monocyte-derived macrophages is correlated with bacterial piliation and hydrophobicity. Infect Immun, 1986. 53(1): p. 207-12.

939

45. Bradley, D.E., Evidence for the retraction of Pseudomonas aeruginosa RNA phage pili. Biochem

940

941 Biophys Res Commun, 1972. 47(1): p. 142-9.

942

943

944

945

46. Laventie, B.J., et al., A Surface-Induced Asymmetric Program Promotes Tissue Colonization by Pseudomonas aeruginosa. Cell Host Microbe, 2019. 25(1): p. 140-152 e6.

47. Shanks, R.M., et al., Saccharomyces cerevisiae-based molecular tool kit for manipulation of genes from gram-negative bacteria. Appl Environ Microbiol, 2006. 72(7): p. 5027-36.

48. Silayeva, O. and A.C. Barnes, Gibson Assembly facilitates bacterial allelic exchange mutagenesis. J Microbiol Methods, 2018. 144: p. 157-163.

948

49. Bachman, J., Site-directed mutagenesis. Methods Enzymol, 2013. 529: p. 241-8.

949

950 aeruginosa biofilm development. Mol Microbiol, 1998. 30(2): p. 295-304.

951

952

953

954

50. O'Toole, G.A. and R. Kolter, Flagellar and twitching motility are necessary for Pseudomonas

51. O'Toole, G.A., Microtiter dish biofilm formation assay. J Vis Exp, 2011(47).

52. Bradford, M.M., A rapid and sensitive method for the quantitation of microgram quantities of protein utilizing the principle of protein-dye binding. Anal Biochem, 1976. 72: p. 248-54.

53. Li, L., et al., Charge Calibration Standard for Atomic Force Microscope Tips in Liquids. Langmuir, 2020. 36(45): p. 13621-13632. 